\title{
ORBITAL PARAMETERS FOR INDUCED AND RESTRICTED REPRESENTATIONS
}

\author{
RONALD L. LIPSMAN
}

\begin{abstract}
General formulas for the spectral decomposition of both induced and restricted representations are laid out for the case of connected Lie groups $H \subset G$. The formulas-which detail the actual spectrum, the multiplicites, and the spectral measure-are in terms of the usual parameters in the so-called orbit method. A proof of these formulas is given in the nilpotent situation. The proof is much simpler than a previously obtained proof using nilpotent algebraic geometry. It is also capable of generalization to nonnilpotent groups. With that in mind, many new examples are presented for semisimple and symmetric homogeneous spaces. Also, a start is made in the case of exponential solvable homogeneous spaces with the treatment of both normal and conormal subgroups.
\end{abstract}

\section{INTRODUCTION}

We are concerned here with one of the basic problems in modern Lie group representations. How do we decompose a general induced representation $\operatorname{Ind}_{H}^{G} \nu$ into irreducible unitary representations of $G$ ? Even in the case that $\nu$ is trivial, there is no general prescription for decomposing the quasi-regular representation $\operatorname{Ind}_{H}^{G} 1$ of $G$ on $L^{2}(G / H)$. If $H$ itself is trivial, we have the regular representation - then a great deal is known, both in the abstract (the Plancherel theorem) and for specific categories of groups $G$. On the other hand, with two exceptions, little progress had been registered until very recently in the study of the general quasi-regular representation. The two exceptions are: first, $H$ a lattice subgroup of $G$ (i.e., $H$ discrete with $G / H$ of finite volume); and second, $G / H$ Riemannian symmetric (i.e., $G$ connected semisimple and $H$ the (compact) stabilizer of a Cartan involution). Attention has focused on general homogeneous spaces in recent years for two reasons. One is the advances made in the study of non-Riemannian symmetric spaces. The second is the relatively new subject of applications of group representations to solvability properties of (invariant) differential equations on groups and homogeneous spaces. The Plancherel theory of the quasi-regular representation is an important tool in those applications.

Received by the editors February $25,1987$.

1980 Mathematics Subject Classification (1985 Revision). Primary 22E27; Secondary 22E45.

Research supported by the National Science Foundation under DMS 84-00900-A02. 
How might one approach the problem of giving a descriptive decomposition of the quasi-regular representation? The answer must be in terms of one of three possible kinds of parameters: semisimple parameters, Mackey machine parameters, or orbit method parameters. For semisimple homogeneous spaces, the former may be the best-but these are unlikely to suggest a recipe for the general case. As with the representation theory of solvable Lie groups, the Mackey machine can be activated to handle most specific nonsemisimple homogeneous spaces, but no general picture emerges this way. The orbit method is the best hope for a general picture. Our basic objective is to give an orbital description of the decomposition of an induced representation $\operatorname{Ind}_{H}^{G} \nu$. Such a decomposition has three components: the spectrum, (i.e., which representations actually occur in the decomposition), their multiplicity, and the spectral measure. We give orbital descriptions of all three, plus an orbital description for restrictions of irreducible representations to a subgroup (see formulas (I) and (R) below). Most of our results in this paper are for nilpotent groups. Ultimately we shall generalize to exponential solvable groups and beyond.

The motivation for formula (I) has existed for 15 years. The orbit method was invented by Kirillov in the early 1960s. But it was the work of Pukanszky [18] and Vergne [21] that gave a good indication of how an orbital formula for an induced representation should look. Unfortunately, this was not really seized upon until recently in the work of Corwin and Greenleaf [2]. They give a formulation for the spectral decomposition in the nilpotent case purely in terms of orbital parameters. Their work is very beautiful and incisive. But there is a difficulty. Their arguments depend critically on the Pukanszky method of stratification of $\mathfrak{g}^{*}$ by layers. This causes two problems-first, a very long and extremely complicated proof of the main formula; and second, the fact that their result is false for exponential solvable groups. Upon seeing [2], I felt that the proof could be simplified and that their formula-suitably altered-was valid for exponential solvable groups and perhaps more.

This paper is the first in a series which will attempt to substantiate these feelings. The foundation of the work is contained in the following two formulas. Let $G$ be exponential solvable, $H \subset G$ a closed connected subgroup. The irreducible unitary representations of $G$ (resp. $H$ ) correspond to $G$-orbits $\mathscr{O} \subset \mathfrak{g}^{*}$ (resp. $H$-orbits $\left.\mathscr{U} \subset \mathfrak{h}^{*}\right)$. The formulas are:

$$
\operatorname{Ind}_{H}^{G} \nu_{\mathscr{U}}=\int_{p^{-1}(\mathscr{U}) / H}^{\oplus} \pi_{\mathscr{C}} d \mu_{G, H}^{\mathscr{U}}(\mathscr{O})=\int_{G \cdot p^{-1}(\mathscr{U}) / G}^{\oplus} n_{\mathscr{O}, \mathscr{U}} \pi_{\mathscr{O}} d \mu_{G, H}^{\mathscr{U}}(\mathscr{O}),
$$

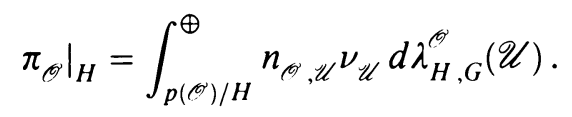

Here $p: \mathfrak{g}^{*} \rightarrow \mathfrak{h}^{*}$ is the canonical projection, the measures are canonically defined (see $\S \S 2-4)$, and $n_{\mathscr{H}, \mathscr{U}}=\# H$-orbits on $\mathscr{O} \cap p^{-1}(\mathscr{U})$. Our main goals in this paper are twofold. The first is to give proofs of these formulas for $G$ nilpotent. As mentioned earlier the proofs in [2,4] (of somewhat different 
formulas) rely totally on "layers," are long and complex, and are completely tied to the nilpotent situation. Ours will be by induction on $\operatorname{dim} G / H$ and have the capability of generalization. I believe a similar method of proof is employed in the thesis of Grélaud [8], but I have never seen it. ${ }^{1}$ The second main goal is to begin the generalization of formulas $(I)$ and $(R)$ to nonnilpotent situations. We move in three directions: (a) a proof of the formulas for $G$ exponential and $H$ normal, (b) a description of $\operatorname{Ind}_{H}^{G} \chi$, when $\chi$ is a character and $H$ is conormal (i.e., $G=H N$ semidirect with $N$ normal), and (c) a presentation of a host of examples to supply clues for nonexponential solvable situations.

The layout of the paper is as follows. It is in two parts-nilpotent (Part I) and nonnilpotent (Part II). Part I begins in $\S 0$ with a statement of the basic results on induced and restricted representations when $H$ is codimension 1 in a nilpotent $G$. (These have been well known since [9].) In $\S 1$ we reformulate the main result of [2]. The reformulated result-Theorem 1.5-is formula (I) for the case that $\nu$ is a character. We prove it in $\S 2$ by induction on $\operatorname{dim} G / H$, the key point being that nilpotence of $G$ ensures the existence of a normal subgroup $N$ of codimension $1, H \subset N \triangleleft G$. In an appendix we prove that the algebraic varieties $\mathscr{O} \cap p^{-1}(\chi)$ are generically smooth manifolds. This allows us to equate the multiplicity function (in the finite case) in formula (I) with the number of components in the intersection $\mathscr{O} \cap p^{-1}(\chi)$. The case $\chi=1$ is separated out in Theorem 2.2. In $\S 3$ we use the fact that the irreducibles are monomial to prove Theorem 1.5 for arbitrary $\nu$. The main result is Theorem 3.1 (see also Theorem 3.5), a completely precise version of formula (I). In $\S 4$ we prove the restriction formula $(R)$ by induction. The precise result is in Theorem 4.2. The last section in Part $I(\S 5)$ gives a new proof of further refinements of the multiplicity function from [2]. Using some real algebraic geometry, it is proved that the multiplicity is either uniformly infinite or bounded finite. (Actually Corwin-Greenleaf obtain a more sophisticated result-namely constant parity of the multiplicity [3] - by employing the theory of semialgebraic sets.) Part II has three sections. In $\S 6$ we prove formulas (I) and (R) for $G$ exponential solvable and $H$ normal (Theorems 6.1,6.2). This is actually used at one point in Part I, but it will also be important for nonnilpotent groups. Another feature of the nonnilpotent case is the potential lack of codimension 1 normal subgroups of $G$ containing $H$. This often occurs, for example, when $H$ is conormal. Such groups are considered in $\S 7$. We decompose the representation induced by a character from a conormal subgroup in terms of Mackey parameters and the oscillator representation. (The reformulation in orbital terms will be presented another time.) The result (see Theorems 7.1, 7.2) is valid for quite general conormal subgroups. The final section $(\S 8)$ presents a variety of nonnilpotent examples, some semisimple and some symmetric. These serve as indicators of

1 The referee has informed me that in Grélaud's thesis (Sur les représentations des groupes de Lie résolubles, Poitiers, 1984-see [8]), slightly different versions of Theorems 1.1 and 6.1 are proved. Also, Example 8(ii), which I originally attributed to Larry Corwin, is due to Grélaud. 
the possibility that formulas (I) and (R) - suitably modified-are true in greater generality.

I would like to conclude the introduction by thanking the referee for several excellent recommendations.

\section{PART I. NilPotent GROUPS}

In this first part we shall consider homogeneous spaces $G / H$ where $G$ is simply connected nilpotent and $H$ is a closed connected subgroup. First we shall reformulate the main result of [2] on the spectrum of $\operatorname{Ind}_{H}^{G} \nu$. It is our revised formula which will generalize out of the nilpotent situation (see Part II). The method of proof we employ is quite different from that of [2]. We shall not use at all the Pukanszky stratification of $\mathfrak{g}^{*}$ by layers. Instead we reason by induction on $\operatorname{dim} G / H$. We begin by setting up the basic Kirillov-Mackey machinery for representations of nilpotent Lie groups.

\section{BASICS}

Let $G$ be a simply connected nilpotent Lie group, $\mathfrak{g}$ its Lie algebra, $\mathfrak{g}^{*}$ the real linear dual. For each $\varphi \in \mathfrak{g}^{*}$, we denote by $\pi_{\varphi}$ the (class of) irreducible unitary representation(s) of $G$ associated to $\varphi$ by the Kirillov orbit method [9]. The map $\varphi \rightarrow \pi_{\varphi}, \mathfrak{g}^{*} \rightarrow \hat{G}$, is $G$-equivariant and factors to a bijection $\mathfrak{g}^{*} / G \rightarrow \hat{G}$. If $H$ is a closed connected subgroup of $G$ with Lie algebra $\mathfrak{h}$, we write $p_{\mathfrak{g}, \mathfrak{h}}: \mathfrak{g}^{*} \rightarrow \mathfrak{h}^{*}$ for the canonical proejction. If $\mathfrak{g}$ and $\mathfrak{h}$ may be understood from the context, we write simply $p: \mathfrak{g}^{*} \rightarrow \mathfrak{h}^{*}$. We set $\mathfrak{h}^{\perp}=p^{-1}(0)$.

Now suppose $\mathfrak{n}$ is an ideal in $\mathfrak{g}$ of codimension $1, N=\exp \mathfrak{n}, p=p_{\mathfrak{g}, \mathfrak{n}}$. The relationship between an irreducible representation of $N$ and those of $G$ that lie over it, together with the corresponding orbit relationships, has long been well understood. We summarize the salient facts in the following theorem (see [9]).

Theorem 0.1. Let $\theta \in \mathfrak{n}^{*}, \gamma_{\theta} \in \hat{N}$ the corresponding representation. Exactly one of the following obtains:

(a) There is a unique G-orbit $\mathscr{O}$ which satisfies $p(\mathscr{O}) \supset N \cdot \theta$. In that case, for any $\varphi \in \mathscr{O} \cap p^{-1}(\theta)$ we have $\pi_{\varphi}=\operatorname{Ind}_{N}^{G} \gamma_{\theta}, \operatorname{dim} G \cdot \varphi=\operatorname{dim} N \cdot \theta+2$, and $p^{-1}(N \cdot \theta)=N \cdot \varphi$.

(b) There is a G-orbit $\mathscr{O}$ which satisfies $p(\mathscr{O}) \supset N \cdot \theta$, but it is not unique. If $\varphi \in \mathscr{O} \cap p^{-1}(\theta)$ and $\alpha \in \mathfrak{n}^{\perp}, \alpha \neq 0$, then the functionals $\{\varphi+t \alpha: t \in \mathbf{R}\}$ lie in distinct orbits $\mathscr{O}_{t}=\mathscr{O}+t \alpha, \operatorname{dim} \mathscr{O}_{t}=\operatorname{dim} N \cdot \theta, p\left(\mathscr{O}_{t}\right)=N \cdot \theta, p^{-1}(N \cdot \theta)=\cup_{t} \mathscr{O}_{t}$, and $\operatorname{Ind}_{N}^{G} \gamma_{\theta}=\int^{\oplus} \pi_{\varphi+t \alpha} d t$.

Conversely, let $\varphi \in \mathfrak{g}^{*}, \pi_{\varphi} \in \hat{G}$ the corresponding representation. Let $\theta=$ $p(\varphi)$. Exactly one of the following obtains:

(a) $\operatorname{dim} N \cdot \theta=\operatorname{dim} G \cdot \varphi-2$. If $X \in \mathfrak{g}, X \notin \mathfrak{n}$, then the functionals $\left\{\theta_{s}=\exp s X \cdot \theta: s \in \mathbf{R}\right\}$ lie in distinct $N$-orbits, $p(G \cdot \varphi)=\bigcup_{s} N \cdot \theta_{s}$, and $\left.\pi_{\varphi}\right|_{N}=\int^{\oplus} \gamma_{\theta_{s}} d s$.

(b) $p(G \cdot \varphi)=N \cdot \theta, \operatorname{dim} G \cdot \varphi=\operatorname{dim} N \cdot \theta$, and $\left.\pi_{\varphi}\right|_{N}=\gamma_{\theta}$. 


\section{Reformulation of Corwin-Greenleaf}

We begin by studying $\operatorname{Ind}_{H}^{G} \chi$, where $\chi$ is a unitary character of the connected subgroup $H$ (inside the simply connected nilpotent group $G$ ). The main result of [2]-when the inducing representation of $H$ is a character-is the following.

Theorem 1.1 (Corwin-Greenleaf). Let $\mathfrak{h}_{\chi}^{\perp}=\left\{\varphi \in \mathfrak{g}^{*}: p(\varphi)=-i d \chi\right\}$. Then

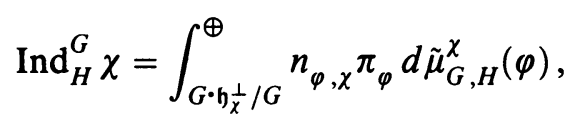

where $G \cdot \mathfrak{h}_{\chi}^{\perp} / G$ is the set of $G$-orbits that meet $p^{-1}(-i d \chi), \tilde{\mu}_{G, H}^{\chi}$ is the pushforward of Lebesgue measure under $\mathfrak{h}_{\chi}^{\perp} \rightarrow G \cdot h_{\chi}^{\perp} / G$, and $n_{\varphi, \chi}$ is given as follows:

if generically on $\mathfrak{h}_{\varphi}^{\perp}$ we have

$$
\begin{array}{ll}
\operatorname{dim} G \cdot \varphi>2 \operatorname{dim} H \cdot \varphi, & \text { then } n_{\varphi, \chi} \equiv+\infty \\
\operatorname{dim} G \cdot \varphi=2 \operatorname{dim} H \cdot \varphi, & \text { then } n_{\varphi, \chi}=\# H \text {-orbits on } G \cdot \varphi \cap \mathfrak{h}_{\chi}^{\perp}, \\
& \text { and is finite. }
\end{array}
$$

In the latter case, the algebraic variety $G \cdot \varphi \cap \mathfrak{h}_{\chi}^{\perp}$ is generically a manifold, so that $n_{\varphi, \chi}=\#$ components of $G \cdot \varphi \cap \mathfrak{h}_{\chi}^{\perp}$.

The push-forward is the measure class obtained by replacing Lebesgue measure on $\mathfrak{h}_{x}^{\perp}$ by an equivalent finite measure and then taking the ordinary image. (Finite measures in that class are often called pseudoimages.) Generically on $\mathfrak{h}_{\chi}^{\perp}$ means aside from a lower-dimensional variety. Although explicit, the formulation of Theorem 1.1 is cumbersome. We reformulate so that the disintegration is specified by a single equation. To do this we need some auxiliary results. First, the map $\mathfrak{h}_{\chi}^{\perp} \rightarrow G \cdot \mathfrak{h}_{\chi}^{\perp} / G$ is $H$-equivariant and factors to a surjective map

$$
P_{\chi}: \mathfrak{h}_{\chi}^{\perp} / H \rightarrow G \cdot \mathfrak{h}_{\chi}^{\perp} / G .
$$

Lemma 1.2. For $\varphi \in \mathfrak{h}_{\chi}^{\perp}$, the number of elements in the fiber $P_{\chi}^{-1}(G \cdot \varphi)$ is exactly

$$
n_{\varphi, \chi}=\# H \text {-orbits in } G \cdot \varphi \cap \mathfrak{h}_{\chi}^{\perp} \text {. }
$$

Proof. This is elementary. If $G$ acts (linearly) on $V$ and $W$ is an $H$-stable subset, then the number of elements in the preimage of an orbit $G \cdot \omega$ under $W / H \rightarrow G \cdot W / G$ is exactly the number of $H$-orbits in $G \cdot \omega \cap W$.

Corollary 1.3. Let $\varphi \in \mathfrak{h}_{\chi}^{\perp}$. Then generically

(i) if $\operatorname{dim} H \cdot \varphi=\operatorname{dim}\left(G \cdot \varphi \cap \mathfrak{h}_{\chi}^{\perp}\right)$, then $n_{\varphi, \chi}=$ \# connected components of $G \cdot \varphi \cap \mathfrak{h}_{\chi}^{\perp}$,

(ii) if $\operatorname{dim} H \cdot \varphi<\operatorname{dim}\left(G \cdot \varphi \cap \mathfrak{h}_{\chi}^{\perp}\right)$, then $n_{\varphi, \chi}=+\infty$. 
Lemma 1.4. Generically on $\mathfrak{h}_{x}^{\perp}$, we have

$$
\operatorname{dim}\left(G \cdot \varphi \cap \mathfrak{h}_{\chi}^{\perp}\right)=\operatorname{dim} G \cdot \varphi-\operatorname{dim} H \cdot \varphi .
$$

Therefore either

(i) $\operatorname{dim} G \cdot \varphi=2 \operatorname{dim} H \cdot \varphi$, equivalently $\operatorname{dim} H \cdot \varphi=\operatorname{dim}\left(G \cdot \varphi \cap \mathfrak{h}_{\chi}^{\perp}\right)$, or

(ii) $\operatorname{dim} G \cdot \varphi>2 \operatorname{dim} H \cdot \varphi$, equivalently $\operatorname{dim} H \cdot \varphi<\operatorname{dim}\left(G \cdot \varphi \cap \mathfrak{h}_{\chi}^{\perp}\right)$.

Proof. Generically on $\mathfrak{h}_{\chi}^{\perp}$, the tangent space to $G \cdot \varphi \cap \mathfrak{h}_{\chi}^{\perp}$ is given at $\varphi$ by $\mathfrak{g} \cdot \varphi \cap \mathfrak{h}^{\perp}$. Therefore ${ }^{2}$

$$
\begin{aligned}
\operatorname{dim}\left(G \cdot \varphi \cap \mathfrak{h}_{\chi}^{\perp}\right) & =\operatorname{dim}\left(\mathfrak{g} \cdot \varphi \cap \mathfrak{h}^{\perp}\right)=\operatorname{dim}\left(\mathfrak{g}_{\varphi}^{\perp} \cap \mathfrak{h}^{\perp}\right) \\
& =\operatorname{dim}\left(\mathfrak{g}_{\varphi}+\mathfrak{h}\right)^{\perp}=\operatorname{dim} \mathfrak{g}-\operatorname{dim}\left(\mathfrak{g}_{\varphi}+\mathfrak{h}\right) \\
& =\operatorname{dim} \mathfrak{g}-\left(\operatorname{dim} \mathfrak{g}_{\varphi}+\operatorname{dim} \mathfrak{h}\right)+\operatorname{dim}\left(\mathfrak{g}_{\varphi} \cap \mathfrak{h}\right) \\
& =\left(\operatorname{dim} \mathfrak{g}-\operatorname{dim} \mathfrak{g}_{\varphi}\right)-\left(\operatorname{dim} \mathfrak{h}-\operatorname{dim} \mathfrak{h}_{\varphi}\right) \\
& =\operatorname{dim} G \cdot \varphi-\operatorname{dim} H \cdot \varphi .
\end{aligned}
$$

We can now use Corollary 1.3 and Lemma 1.4 to simplify the statement of Theorem 1.1.

Theorem 1.5. We have

$$
\operatorname{Ind}_{H}^{G} \chi=\int_{\mathfrak{h}_{\grave{1}}^{1} / H}^{\oplus} \pi_{\varphi} d \mu_{G, H}^{\chi}(\varphi),
$$

where $\mu_{G, H}^{\chi}$ is the push-forward of Lebesgue measure under $\mathfrak{h}_{\chi}^{\perp} \rightarrow \mathfrak{h}_{\chi}^{\perp} / H$.

Now a general principle of direct integral theory says the following: let $G$ be a separable type I group and

$$
\int_{X}^{\oplus} \pi_{x} d \mu(x)
$$

a direct integral of irreducible unitary representations. In particular $x \rightarrow \pi_{x}$, $X \rightarrow \operatorname{Irr}(G)$, is a Borel injection ( $\mu$-a.e.). If we write $x_{1} \sim x_{2}$ to mean $\pi_{x_{1}}$ is unitarily equivalent to $\pi_{x_{2}}$ and set $Y=X / \sim$, it follows that

$$
\int_{X}^{\oplus} \pi_{x} d \mu(x) \simeq \int_{Y}^{\oplus} n_{y} \pi_{y} d \bar{\mu}(y)
$$

when $\bar{\mu}$ is the push-forward of $\mu$ under the canonical projection $p: X \rightarrow Y$ and $n_{y}=\# p^{-1}(y)$. In fact, $(Y, \bar{\mu})$ is countably separated (since $G$ is type $\mathrm{I}$ ) and the equality results immediately from the disintegration of $(X, \mu)$ under $p$. (For a reference see [10, Theorem 2.1], or more precisely reference [3]

\footnotetext{
${ }^{2}$ The referee has pointed out that I am being somewhat disingenuous when I assert complete avoidance of layers in my arguments. In one point at least I have obscured their appearance, namely in the assertion that, generically, the orbit intersections $G \cdot \varphi \cap \mathfrak{h}_{\chi}^{\frac{1}{2}}(\mathfrak{g})$ have the same dimension as the tangent spaces $\mathfrak{g} \cdot \varphi \cap \mathfrak{h}^{\perp}$. A very general result along these lines is given in the Appendix to $\S 1$. Its proof depends critically on the Chevalley-Pukanszky parametrization of the orbits and orbit space for a unipotent group action.
} 
therein (Bourbaki, Intégration, Chapitre VI) since $\sim$ does not correspond to a group action.)

According to the corollary and lemma cited, it follows automatically from Theorem 1.5 that

$$
\operatorname{Ind}_{H}^{G} \chi=\int_{G \cdot \mathfrak{h} \frac{1}{\chi} / G}^{\oplus} n_{\varphi, \chi} \pi_{\varphi} d \tilde{\mu}_{G, H}^{\chi}(\varphi),
$$

where $n_{\varphi, \chi}=\# H$-orbits in $G \cdot \varphi \cap \mathfrak{h}_{\chi}^{\perp}=\#$ connected components of $G \cdot \varphi \cap \mathfrak{h}_{\chi}^{\perp}$, in the equal dimension case. We shall always use the phrase "equal dimension case" to refer to the situation described by Lemma 1.4(i). Note also that in the equal dimension case, our ability to identify $n_{\varphi, \chi}$ with \# connected components requires knowing the $G \cdot \varphi \cap \mathfrak{h}_{\chi}^{\perp}$ is generically a manifold. We prove this in an appendix to the next section.

Realizing the decomposition of $\operatorname{Ind}_{H}^{G} \chi$ as in Theorem 1.5 instead of Theorem 1.1 effects more than a verbal or symbolic simplification of the multiplicity formula. The formulation of Theorem 1.5 allows for generalization to nonnilpotent groups-Theorem 1.1 does not.

Example 1.6. Let $\mathfrak{g}$ be the 3-dimensional exponential solvable Lie algebra with generators $T, X, Y$ satisfying the relations $[T, X]=X+Y,[T, Y]=-X+Y$. Let $\mathfrak{h}=\mathbf{R} X$. Then generically on $\mathfrak{h}^{\perp}, \operatorname{dim} G \cdot \varphi=2=2 \operatorname{dim} H \cdot \varphi$, but $\operatorname{Ind}_{H}^{G} 1$ is of uniform infinite multiplicity. So Theorem 1.1 is false.

This example was shown to me by Larry Corwin as evidence that Theorem 1.1 is not true for exponential solvable groups. ${ }^{3}$ That contradicted my intuition, and caused me to seek a reformulation of the result. Indeed Theorem 1.5 is true for Example 1.6. The point is that for nilpotent groups, the coadjoint action is algebraic. Therefore in the equal dimension case, the number of components must be finite (see $\S 5$ and Remark 2.1(ii)). That is not so for exponential solvable groups. The equal dimension case allows for infinitely many components and so infinite multiplicity in Theorem 1.5.

I take as my working hypothesis that Theorem 1.5 is true, at the very least for exponential solvable groups (see Part II).

\section{1a. APPENDIX}

In this paper, in the proof of Theorems $1.5,3.5$, and 4.2 , we have used implicitly the following proposition. The proof is adapted from arguments in [2 and 4] and especially from a new preprint of Corwin-Greenleaf (A canonical approach to multiplicity formulas for induced and restricted representations of nilpotent Lie groups.)

\footnotetext{
${ }^{3}$ The referee has informed me that in Gréland's thesis (Sur les représentations des groupes de Lie résolubles Poitiers, 1984-see [8]), slightly different versions of Theorems 1.1 and 6.1 are proved. Also, Example 8(ii), which I originally attributed to Larry Corwin, is due to Grélaud.
} 
Proposition 1.7. Let $H \subset N \triangleleft G$ be simply connected nilpotent Lie groups. Fix $\psi \in \mathfrak{h}^{*}$. Then, generically on $p_{\mathfrak{n}, \mathfrak{h}}^{-1}(H \cdot \psi)$, we have $G \cdot \theta \cap p_{\mathfrak{n}, \mathfrak{h}}^{-1}(H \cdot \psi)$ has the same dimension as $\mathfrak{g} \cdot \theta \cap p_{\mathfrak{n}, \mathfrak{h}}^{-1}(\mathfrak{h} \cdot \psi)$.

Proof. For convenience write $p=p_{\mathrm{n}, \mathfrak{h}}$ and $\mathscr{O}=H \cdot \psi$. Now the action of $G$ on $\mathfrak{n}^{*}$ is unipotent algebraic. It is well known that there is a $G$-invariant Zariski-open subset $\mathscr{U} \subset \mathrm{n}^{*}$ such that $\mathscr{U} \cap p^{-1}(\mathscr{O})$ is Zariski-open in $p^{-1}(\mathscr{O})$, and a nonsingular birational map

$$
\Phi: \mathscr{U} \rightarrow \Sigma \times V,
$$

where $\Sigma$ is a Zariski-open subset of affine space, $V$ is affine and

$$
\begin{aligned}
& \Phi^{-1}\{(\sigma, v): v \in V\} \text { constitutes a } G \text {-orbit, for each fixed } \sigma \in \Sigma, \\
& \Phi^{-1}(\sigma, v) \text { and } \Phi^{-1}\left(\sigma^{\prime}, v^{\prime}\right) \text { lie in the same } G \text {-orbit } \Leftrightarrow \sigma=\sigma^{\prime} .
\end{aligned}
$$

\section{Consider}

$$
f: \mathscr{U} \cap p^{-1}(\mathscr{O}) \rightarrow \Sigma
$$

defined by

$$
f=\left.p_{\Sigma} \circ \Phi\right|_{\mathscr{U} \cap p^{-1}(0)} .
$$

We restrict attention to the Zariski-open subset $\mathscr{U}_{1} \subset \mathscr{U} \cap p^{-1}(\mathscr{O})$ on which $f$ has maximal rank. On $\mathscr{U}_{1}, f$ determines a foliation in which the leaves are given by the orbit intersections $G \cdot \theta \cap p^{-1}(\mathscr{O}) \quad\left(\theta \in \mathscr{U}_{1} \subset \mathfrak{n}^{*}\right)$. These have dimension equal to $\operatorname{dim} \operatorname{ker}(d f)_{\theta}$, for generic points. Corwin and Greenleaf, in their preprint, actually compute the latter (in a more special situation). They obtain

$$
\operatorname{ker}(d f)_{\theta}=\mathfrak{g} \cdot \theta \cap p^{-1}(\mathfrak{h} \cdot \psi) .
$$

In fact, it is clear that the tangent space to the orbit intersection is contained in

$$
T_{\theta}(G \cdot \theta) \cap T_{\theta}\left(p^{-1}(\mathscr{O})\right)=\mathfrak{g} \cdot \theta \cap p^{-1}(\mathfrak{h} \cdot \psi) .
$$

But conversely, since $f$ is a restriction of the projection $p_{\Sigma} \circ \Phi$, it must be that

$$
\begin{aligned}
\operatorname{ker}(d f)_{\theta} & \supset \operatorname{ker} d\left(p_{\Sigma} \circ \Phi\right) \cap T_{\theta}\left(p^{-1}(\mathscr{O})\right) \\
& =\operatorname{ker} d\left(p_{\Sigma} \circ \Phi\right) \cap p^{-1}(\mathfrak{h} \cdot \psi) .
\end{aligned}
$$

And finally, since $p_{\Sigma} \circ \Phi$ is constant on $G \cdot \theta \subset \mathscr{U}$, we must have

$$
\operatorname{ker} d\left(p_{\Sigma} \circ \Phi\right) \supset T_{\theta}(G \cdot \theta)=\mathfrak{g} \cdot \theta .
$$

This completes the proof.

\section{ORBITAL DECOMPOSITION of $\operatorname{Ind}_{H}^{G} \chi$}

The object of this section is to prove Theorem 1.5 when $G$ is simply connected nilpotent, $H$ closed connected. The argument is by induction on $\operatorname{dim} G / H$. 
$\operatorname{dim} G / H=1$. Since $G$ is nilpotent, this forces $H$ to be normal. In that situation, Theorem 1.5 becomes a special case of Theorem 6.1. That theorem is placed in Part II because it is valid for exponential solvable groups; its proof does not depend on anything from Part I.

$\operatorname{dim} G / H>1$. Once again we invoke the nilpotency of $G$, this time to assert that we may find a closed connected normal subgroup $N$ of $G$ with $H \subset N \triangleleft G$ and $\operatorname{dim} G / N=1$. By the induction hypothesis, Theorem 1.5 is true for $\operatorname{Ind}_{H}^{N} \chi$. To avoid ambiguity in the coming proof we employ the following notational scheme. Write

$$
\begin{aligned}
& \mathfrak{h}_{\chi}^{\perp}(\mathfrak{g})=\left\{\varphi \in \mathfrak{g}^{*}: p_{\mathfrak{g}, \mathfrak{h}}(\varphi)=\varphi \mid \mathfrak{h}=-i d \chi\right\}, \\
& \mathfrak{h}_{\chi}^{\perp}(\mathfrak{n})=\left\{\theta \in \mathfrak{n}^{*}: p_{\mathfrak{n}, \mathfrak{h}}(\theta)=\theta \mid \mathfrak{h}=-i d \chi\right\} .
\end{aligned}
$$

Then by induction in stages, the induction hypothesis, and the fact that direct integrals commute with induction, we have the first three equalities in the following chain:

$$
\begin{aligned}
\operatorname{Ind}_{H}^{G} \chi & =\operatorname{Ind}_{N}^{G} \operatorname{Ind}_{H}^{N} \chi=\operatorname{Ind}_{N}^{G} \int_{\mathfrak{h}_{x}^{\frac{1}{x}}(\mathfrak{n}) / H}^{\oplus} \gamma_{\theta} d \mu_{N, H}^{\chi}(\theta) \\
& =\int_{\mathfrak{h}_{x}^{\left.\frac{1}{(n)}\right) / H}}^{\oplus} \operatorname{Ind}_{N}^{G} \gamma_{\theta} d \mu_{N, H}^{\chi}(\theta)=\int_{\mathfrak{h}_{x}^{\frac{1}{x}(\mathfrak{g}) / H}}^{\oplus} \pi_{\varphi} d \mu_{G, H}^{\chi}(\varphi) .
\end{aligned}
$$

We must prove the last equation, labeled (A). We shall do it by examining four separate cases. Let $\varphi \in \mathfrak{h}_{\chi}^{\perp}(\mathfrak{g}), \theta=\varphi \mid \mathfrak{n} \in \mathfrak{h}_{\chi}^{\perp}(\mathfrak{n})$. Then we have a pair of dichotomous situations: Either (i) $\operatorname{dim} G \cdot \varphi=2 \operatorname{dim} H \cdot \varphi$ or (ii) $\operatorname{dim} G \cdot \varphi>$ $2 \operatorname{dim} H \cdot \varphi$; and either (a) $\operatorname{Ind}_{N}^{G} \gamma_{\theta}$ is irreducible or (b) $\operatorname{Ind}_{N}^{G} \gamma_{\theta}=\int^{\oplus} \pi_{\varphi+t \alpha} d t$, $\alpha \in \mathfrak{n}^{\perp}$.

Of course we do not look at arbitrary $\varphi, \theta$. Both of the dichotomous situations above are valid generically. More precisely, there are Zariski-open subsets $\mathscr{U}_{1}, \mathscr{U}_{2}$ of $\mathfrak{h}_{\chi}^{\perp}(\mathfrak{g}), \mathfrak{h}_{\chi}^{\perp}(\mathfrak{n})$ (resp.) such that either (i) or (ii), resp. (a) or (b), holds. In the following we consider only

$$
\varphi \in \mathscr{U}_{1} \cap p_{\mathfrak{g}, \mathfrak{n}}^{-1}\left(\mathscr{U}_{2}\right), \quad \theta \in \mathscr{U}_{2} \cap p_{\mathfrak{g}, \mathfrak{n}}\left(\mathscr{U}_{1}\right) .
$$

Next we observe that the representations on the two sides of equation (A) are quasi-equivalent. This is fairly evident by a mixture of Kirillov and Mackey. On the right side, the spectrum consists of those irreducible representations of $G$ whose orbits meet the closed variety $\mathfrak{h}_{\chi}^{\perp}(\mathfrak{g})$. On the left side the spectrum consists of those irreducible representations of $G$ which lie over the representations of $N$ whose orbits meet $\mathfrak{h}^{\perp}(\mathfrak{n})$. But $p_{\mathfrak{g}, \mathfrak{n}}^{-1}\left(\mathfrak{h}_{\chi}^{\perp}(\mathfrak{n})\right)=\mathfrak{h}_{\chi}^{\perp}(\mathfrak{g})$. Thus it is clear (e.g., from [7]) -and in fact is implicit in the assertions of Kirillov in [9]) that these two spectra are the same. What is not at all evident is that the multiplicities agree.

Before attending to the multiplicity, we should also observe that the measure classes on both sides of equation (A) are in agreement. To see this we must distinguish between cases (a) and (b). First of all, every group action is unipotent, 
so algebraic. Thus all quotient spaces are countably separated. Next it is clear from the commutative diagram

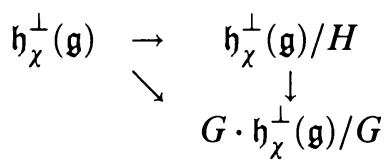

that the push-forward of the Lebesgue measure class on $\mathfrak{h}_{\chi}^{\perp}(\mathfrak{g})$ to $\mathfrak{h}_{\chi}^{\perp}(\mathfrak{g}) / H$ projects down to the push-forward of Lebesgue measure on $G \cdot \mathfrak{h}_{\chi}^{\perp}(\mathfrak{g}) / G$. The same is true with $G$ replaced by $N$. In case (a) the map $\gamma_{\theta} \rightarrow \operatorname{Ind}_{N}^{G} \gamma_{\theta}$ injects (the generic part of) $\hat{N} / G$ into $\hat{G}$ and carries $\mu_{N, H}^{\chi}$ into a push-forward on $\hat{G}$. It agrees with $\mu_{G, H}^{\chi}$ because it is also the push-forward of Lebesgue measure on $\mathfrak{h}_{\chi}^{\perp}(\mathfrak{n})$ under $\mathfrak{h}_{\chi}^{\perp}(\mathfrak{n}) \rightarrow G \cdot \mathfrak{h}_{\chi}^{\perp}(\mathfrak{n}) / G$, which, in case $(\mathrm{a})$, is identified to $G \cdot \mathfrak{h}_{\chi}^{\perp}(\mathfrak{g}) / G$ (generically). (See Remark 2.1 below.) In case (b) the representations $\operatorname{Ind}_{N}^{G} \gamma_{\theta}$ decompose according to Theorem 0.1. Clearly the fiber measure $d t d \theta$ over Lebesgue measure on $\mathfrak{h}_{\chi}^{\perp}(\mathfrak{n})$

$$
\begin{aligned}
\mathfrak{n}^{\perp} \rightarrow & \mathfrak{h}_{\chi}^{\perp}(\mathfrak{g}) \\
\downarrow & \\
& \mathfrak{h}_{\chi}^{\perp}(\mathfrak{n})
\end{aligned}
$$

agrees with Lebesgue measure on $\mathfrak{h}_{\chi}^{\perp}(\mathfrak{g})$. Thus it is also evident that the fiber measure $d t d \mu_{N, H}^{\chi}$ agrees with $d \mu_{G, H}^{\chi}$.

We turn finally to our case-by-case verification of equal multiplicity in equation (A).

(iia) In this case we show that both sides of equation (A) have uniform infinite multiplicity. We take $\varphi \in h_{\chi}^{\perp}(\mathfrak{g}), \theta=\left.\varphi\right|_{\mathfrak{n}} \in h_{\chi}^{\perp}(\mathfrak{n})$, both generic as explained above. In case (a) we have

$$
\pi_{\varphi}=\operatorname{Ind}_{N}^{G} \gamma_{\theta}
$$

Consider

$$
S=\left\{g \in G: g \cdot \varphi \in \mathfrak{h}_{\chi}^{\perp}(\mathfrak{g})\right\} .
$$

$S$ is a closed submanifold of $G$, not a subgroup in general. Clearly $H \subset S$. The map

$$
S \rightarrow G \cdot \varphi \cap \mathfrak{h}_{\chi}^{\perp}(\mathfrak{g}), \quad s \rightarrow s \cdot \varphi,
$$

is a submersion. Moreover

$$
\operatorname{dim} H \cdot \varphi<\operatorname{dim} S \cdot \varphi
$$

(by hypothesis (ii) and Lemma 1.4). All the representations $\pi_{s \cdot \varphi}, s \in S$, are equivalent. Therefore $\int_{\mathfrak{h}_{x}^{\frac{1}{x}}(\mathfrak{g}) / H}^{\oplus} \pi_{\varphi}$ is of uniform infinite multiplicity.

We have to show the left side of (A) also is of uniform infinite multiplicity. Clearly, by the same technique, we are done if we can prove that

$$
\operatorname{dim} G \cdot \theta \cap \mathfrak{h}_{\chi}^{\perp}(\mathfrak{n})>\operatorname{dim} H \cdot \theta .
$$


Now by a standard fact [19, Lemma 2, or 11, p. 271, (iv)], we have $N_{\theta}$. $\varphi=\varphi+\left(\mathfrak{g}_{\theta}+\mathfrak{n}\right)^{\perp}$. But $N=G_{\gamma}=G_{\theta} N \Rightarrow G_{\theta} \subset N$; that is, $G_{\theta}=N_{\theta}$. Hence $\operatorname{dim} N_{\theta} / N_{\varphi}=1$. Furthermore $G_{\varphi}=\left(G_{\theta}\right)_{\varphi}=\left(N_{\theta}\right)_{\varphi}=N_{\varphi}$. Hence $\operatorname{dim} G_{\theta} / G_{\varphi}=1$ also. In particular $\operatorname{dim} G \cdot \theta=\operatorname{dim} G \cdot \varphi-1 . \operatorname{But} \operatorname{dim} G \cdot \varphi>$ $2 \operatorname{dim} H \cdot \varphi$ and both are even. Therefore $\operatorname{dim} G \cdot \theta>2 \operatorname{dim} H \cdot \varphi$. Of course $\mathfrak{h}_{\varphi} \subset \mathfrak{h}_{\theta}$, so $\operatorname{dim} H \cdot \varphi \geq \operatorname{dim} H \cdot \theta$.

Next we claim that $\mathfrak{g} \cdot \theta=\mathfrak{n}_{\varphi}^{\perp}$. (Here, and in the rest of the argument, a perp sign $(\perp)$ without modifier means $\perp$ in $\mathfrak{n}^{*}$.) Indeed $\mathfrak{g} \cdot \theta\left(\mathfrak{n}_{\varphi}\right)=\theta\left[\mathfrak{g}, \mathfrak{n}_{\varphi}\right]=$ $\varphi\left[\mathfrak{g}, \mathfrak{n}_{\varphi}\right]=0$; that is, $\mathfrak{g} \cdot \theta \subset \mathfrak{n}_{\varphi}^{\perp}$. But $\operatorname{dim} \mathfrak{g} \cdot \theta=\operatorname{dim} \mathfrak{g} / \mathfrak{g}_{\theta}=\operatorname{dim} \mathfrak{n} / \mathfrak{n}_{\theta}+1=$ $\operatorname{dim} \mathfrak{n} / \mathfrak{n}_{\varphi}=\operatorname{dim} \mathfrak{n}_{\varphi}^{\perp}$. Now in analogy with our earlier observation in the proof of Lemma 1.4, the tangent space to $G \cdot \theta \cap \mathfrak{h}_{\chi}^{\perp}(\mathfrak{n})$ has the dimension of the space $\mathfrak{g} \cdot \theta \cap \mathfrak{h}^{\perp}$ (generically). But

$$
\mathfrak{g} \cdot \theta \cap \mathfrak{h}^{\perp}=\mathfrak{n}_{\varphi}^{\perp} \cap \mathfrak{h}^{\perp}=\left(\mathfrak{n}_{\varphi}+\mathfrak{h}\right)^{\perp} .
$$

Therefore

$$
\begin{aligned}
\operatorname{dim} G \cdot \theta \cap \mathfrak{h}_{\chi}^{\perp}(\mathfrak{n}) & =\operatorname{dim} \mathfrak{g} \cdot \theta \cap \mathfrak{h}^{\perp}=\operatorname{dim} \mathfrak{n} /\left(\mathfrak{n}_{\varphi}+\mathfrak{h}\right) \\
& =\left(\operatorname{dim} \mathfrak{n}-\operatorname{dim} \mathfrak{n}_{\varphi}\right)-\left(\operatorname{dim} \mathfrak{h}-\operatorname{dim} \mathfrak{h}_{\varphi}\right) \\
& =\operatorname{dim} \mathfrak{n} / \mathfrak{n}_{\varphi}-\operatorname{dim} \mathfrak{h} \cdot \varphi \\
& =\operatorname{dim} \mathfrak{g} \cdot \theta-\operatorname{dim} \mathfrak{h} \cdot \varphi \\
& >\operatorname{dim} \mathfrak{h} \cdot \varphi \\
& \geq \operatorname{dim} \mathfrak{h} \cdot \theta .
\end{aligned}
$$

(See footnote 2 and Proposition 1.7.) That completes the argument in this case.

(iib) We shall prove that, as in (iia), both sides of equation (A) have uniform infinite multiplicity. We fix $\alpha \in \mathfrak{g}^{*},\left.\alpha\right|_{\mathfrak{n}}=0, \alpha \neq 0$. Also, as before, we write $\varphi \in \mathfrak{h}_{\chi}^{\perp}(\mathfrak{g}), \theta=\left.\varphi\right|_{\mathfrak{n}} \in \mathfrak{h}_{\chi}^{\perp}(\mathfrak{n})$, both generic. We know by Theorem 0.1 that the functionals $\varphi+t \alpha, t \in \mathbf{R}$, lie in distinct orbits and

$$
\operatorname{Ind}_{N}^{G} \gamma_{\theta}=\int^{\oplus} \pi_{\varphi+t \alpha} d t \text {. }
$$

We make several easy observations. First $\left.g \cdot \alpha\right|_{\mathfrak{n}}=0, \forall g \in G$. Next we note that $\varphi \in \mathfrak{h}_{\chi}^{\perp}(\mathfrak{g}) \Rightarrow \varphi+t \alpha \in \mathfrak{h}_{\chi}^{\perp}(\mathfrak{g}), \forall t \in \mathbf{R}$. Now exactly as in case (iia) we have $\pi_{s \cdot \varphi}=\pi_{\varphi}, s \in S=\left\{\mathfrak{g} \in G: \mathfrak{g} \cdot \varphi \in \mathfrak{h}_{\chi}^{\perp}(\mathfrak{g})\right\}$ and $\operatorname{dim} H \cdot \varphi<\operatorname{dim} S \cdot \varphi$. So we have infinite multiplicity on the right side of equation (A). But on the left side we know that the induced representations that appear in the direct integral break up into inequivalent irreducible representations. Therefore to show infinite multiplicity, we must establish (as before) that

$$
\operatorname{dim} G \cdot \theta \cap \mathfrak{h}_{\chi}^{\perp}(\mathfrak{n})>\operatorname{dim} H \cdot \theta .
$$

This time we have $\mathfrak{g} \cdot \theta=\mathfrak{n}_{\theta}^{\perp}$ (recall all unattended perps are in $\mathfrak{n}^{*}$ ). Indeed the inclusion $\mathfrak{g} \cdot \theta \subset \mathfrak{n}_{\theta}^{\perp}$ follows from $\mathfrak{g} \cdot \theta\left(\mathfrak{n}_{\theta}\right)=\theta\left[\mathfrak{g}, \mathfrak{n}_{\theta}\right]=0$. This uses the 
equality $\mathfrak{n}_{\theta}=\mathfrak{n}_{\varphi}$ which follows from $N_{\theta} \cdot \varphi=\varphi+\left(\mathfrak{g}_{\theta}+\mathfrak{n}\right)^{\perp}=\varphi$ (since in case (b), $\left.G=G_{\gamma}=G_{\theta} N\right)$. Note also that $G / N=G_{\theta} / N_{\theta}$. Therefore

$$
\operatorname{dim} \mathfrak{g}_{\theta}=\operatorname{dim} \mathfrak{n}_{\theta}+1 .
$$

We also have $\operatorname{dim} G \cdot \varphi=\operatorname{dim} N \cdot \theta \Rightarrow \operatorname{dim} \mathfrak{g} / \mathfrak{g}_{\varphi}=\operatorname{dim} \mathfrak{n} / \mathfrak{n}_{\theta}$. Hence we have the following string of equalities:

$$
\begin{aligned}
& \operatorname{dim} \mathfrak{g}-\operatorname{dim} \mathfrak{g}_{\varphi}=\operatorname{dim} \mathfrak{n}-\operatorname{dim} \mathfrak{n}_{\theta} ; \\
& 1+\operatorname{dim} \mathfrak{n}-\operatorname{dim} \mathfrak{g}_{\varphi}=\operatorname{dim} \mathfrak{n}-\operatorname{dim} \mathfrak{n}_{\theta} ; \\
& \operatorname{dim} \mathfrak{g}_{\theta}=1+\operatorname{dim} \mathfrak{n}_{\theta}=\operatorname{dim} \mathfrak{g}_{\varphi} .
\end{aligned}
$$

That is, $\mathfrak{g}_{\varphi}=\mathfrak{g}_{\theta}$. Thus

$$
\operatorname{dim} \mathfrak{g} \cdot \theta=\operatorname{dim} \mathfrak{g} / \mathfrak{g}_{\theta}=\operatorname{dim} \mathfrak{g} / \mathfrak{g}_{\varphi}=\operatorname{dim} \mathfrak{n} / \mathfrak{n}_{\theta}=\operatorname{dim} \mathfrak{n}_{\theta}^{\perp} .4
$$

Finally,

$$
\begin{aligned}
\operatorname{dim} \mathfrak{g} \cdot \theta \cap \mathfrak{h}^{\perp} & =\operatorname{dim} \mathfrak{n}_{\theta}^{\perp} \cap \mathfrak{h}^{\perp}=\operatorname{dim} \mathfrak{n} /\left(\mathfrak{n}_{\theta}+\mathfrak{h}\right) \\
& =\left(\operatorname{dim} \mathfrak{n}-\operatorname{dim} \mathfrak{n}_{\theta}\right)-\left(\operatorname{dim} \mathfrak{h}-\operatorname{dim} \mathfrak{h}_{\theta}\right) \\
& =\operatorname{dim} \mathfrak{n} \cdot \theta-\operatorname{dim} \mathfrak{h} \cdot \theta \\
& =\operatorname{dim} \mathfrak{g} \cdot \varphi-\operatorname{dim} \mathfrak{h} \cdot \theta \\
& >2 \operatorname{dim} \mathfrak{h} \cdot \varphi-\operatorname{dim} \mathfrak{h} \cdot \theta \\
& \geq \operatorname{dim} \mathfrak{h} \cdot \theta .
\end{aligned}
$$

As in case (a) this completes the argument. Thus the proof in the unequal dimension case is done. We have infinite multiplicity (regardless of (a) or (b)). Now we pass to the equal dimension case, i.e., (i). This time we shall have finite multiplicity (see Remark 2.1(ii)). We must distinguish between cases (a) and (b) again. The latter turns out to be easier, so we present it first.

(ib) We start as usual with $\varphi \in \mathfrak{h}_{\chi}^{\perp}(\mathfrak{g}), \theta=\left.\varphi\right|_{\mathfrak{n}} \in \mathfrak{h}_{\chi}^{\perp}(\mathfrak{n})$, both generic. The assumptions are $\operatorname{dim} G \cdot \varphi \cap \mathfrak{h}_{\chi}^{\perp}(\mathfrak{g})=\operatorname{dim} H \cdot \varphi$ and

$$
\operatorname{Ind}_{N}^{G} \gamma_{\theta}=\int^{\oplus} \pi_{\varphi+t \alpha} d t
$$

$\alpha \in \mathfrak{n}^{\perp}, \alpha \neq 0$. We know that the multiplicity is finite on the right side of equation (A); in fact, it equals the \#H-orbits in $G \cdot \varphi \cap \mathfrak{h}_{\chi}^{\perp}(\mathfrak{g})$ (Remark 2.1(ii)). We first observe that the multiplicity is finite on the left side also. Indeed, we already know (from examining case (b) in situation (ii)) that

$$
\mathfrak{g}_{\varphi}=\mathfrak{g}_{\theta}, \quad \mathfrak{n}_{\varphi}=\mathfrak{n}_{\theta}, \quad \text { and } \mathfrak{g} \cdot \theta=\mathfrak{n}_{\theta}^{\perp} .
$$

${ }^{4}$ The referee has suggested a much simpler argument, namely that $\mathfrak{g}=\mathfrak{g}_{\theta}+\mathfrak{n}$ implies $\mathfrak{g} \cdot \theta=$ $\mathfrak{n} \cdot \theta$, which implies $\mathfrak{g} \cdot \theta=\mathrm{n}_{\theta} \frac{1}{\text {, since }} \mathrm{n} \cdot \theta=\mathrm{n}_{\theta} \frac{1}{\theta}$ is evident. I have left in my argument since I also need the intermediate fact $\mathfrak{g}_{\varphi}=\mathfrak{g}_{\theta}$ later on. 
Therefore $\mathfrak{h}_{\theta}=\mathfrak{h} \cap \mathfrak{n}_{\theta}=\mathfrak{h} \cap \mathfrak{n}_{\varphi}=\mathfrak{h}_{\varphi}$. Moreover

$$
\begin{aligned}
\operatorname{dim} \mathfrak{g} \cdot \theta \cap \mathfrak{h}^{\perp} & =\operatorname{dim} \mathfrak{n}_{\theta}^{\perp} \cap \mathfrak{h}^{\perp} \\
& =\operatorname{dim} \mathfrak{n} \cdot \theta-\operatorname{dim} \mathfrak{h} \cdot \theta \\
& =\operatorname{dim} \mathfrak{g} \cdot \varphi-\operatorname{dim} \mathfrak{h} \cdot \theta \\
& =2 \operatorname{dim} \mathfrak{h} \cdot \varphi-\operatorname{dim} \mathfrak{h} \cdot \theta \\
& =\operatorname{dim} \mathfrak{h} \cdot \theta .
\end{aligned}
$$

Thus, as indicated, the multiplicity is finite (Remark 2.1(ii)), and obviously-by the general principle enunciated in Lemma $1.4-$ it is

$$
\# H \text {-orbits in } G \cdot \theta \cap \mathfrak{h}_{\chi}^{\perp}(\mathfrak{n}) \text {. }
$$

Hence to show equality of multiplicity, it is enough to prove that the natural map

$$
G \cdot \varphi \cap \mathfrak{h}_{\chi}^{\perp}(\mathfrak{g}) \rightarrow G \cdot \theta \cap \mathfrak{h}_{\chi}^{\perp}(\mathfrak{n}),
$$

which is $H$-equivariant, is a bijection. That it is a surjection is completely obvious. But it is also injective. Indeed if $\left.g_{1} \cdot \varphi\right|_{\mathfrak{n}}=\left.g_{2} \cdot \varphi\right|_{\mathfrak{n}}$, then $g_{2}^{-1} g_{1} \cdot \theta=$ $\theta \Rightarrow g_{2}^{-1} g_{1} \in G_{\theta}=G_{\varphi}$.

We come finally to the most complicated case.

(ia) This time, starting with $\varphi \in \mathfrak{h}_{\chi}^{\perp}(\mathfrak{g}), \theta=\left.\varphi\right|_{\mathfrak{n}} \in \mathfrak{h}^{\perp} \chi(\mathfrak{n})$ both generic, we have $\operatorname{dim} G \cdot \varphi \cap \mathfrak{h}_{\chi}^{\perp}(\mathfrak{g})=\operatorname{dim} H \cdot \varphi$ and

$$
\pi_{\varphi}=\operatorname{Ind}_{N}^{G} \gamma_{\theta} \text {. }
$$

Of course the multiplicity on the right side of equation $(A)$ is finite and equals

$$
\# H \text {-orbits on } G \cdot \varphi \cap_{\chi}^{\perp}(\mathfrak{g}) \text {. }
$$

As in (ib) we first show that the multiplicity on the left side is finite. We begin by observing that

$$
\begin{aligned}
2 \operatorname{dim} H \cdot \theta & \leq \operatorname{dim} N \cdot \theta \\
& =\operatorname{dim} G \cdot \varphi-2 \\
& =2(\operatorname{dim} H \cdot \varphi-1) .
\end{aligned}
$$

Therefore $\operatorname{dim} H \cdot \theta \leq \operatorname{dim} H \cdot \varphi-1$. But the codimension of $\mathfrak{h}_{\varphi}$ in $\mathfrak{h}_{\theta}$ is at most 1 , since $\operatorname{dim} \mathfrak{g} / \mathfrak{n}=1$. Therefore

$$
\operatorname{dim} H \cdot \theta=\operatorname{dim} H \cdot \varphi-1 \text {. }
$$

Next we compute

$$
\begin{aligned}
\operatorname{dim} \mathfrak{g} \cdot \theta \cap \mathfrak{h}^{\perp} & =\operatorname{dim} \mathfrak{n}_{\varphi}^{\perp} \cap \mathfrak{h}^{\perp} \\
& =\operatorname{dim} \mathfrak{n} \cdot \varphi-\operatorname{dim} \mathfrak{h} \cdot \varphi \\
& =\operatorname{dim} \mathfrak{g} \cdot \varphi-1-\operatorname{dim} \mathfrak{h} \cdot \varphi \\
& =\operatorname{dim} \mathfrak{h} \cdot \varphi-1 \\
& =\operatorname{dim} \mathfrak{h} \cdot \theta .
\end{aligned}
$$


Therefore

$$
\operatorname{dim} G \cdot \theta \cap \mathfrak{h}_{\chi}^{\perp}(\mathfrak{n})=\operatorname{dim} H \cdot \theta,
$$

and so the multiplicity on the left side of (A) is finite (Remark 2.1(ii)). The general principle of Lemma 1.4 guarantees that it is

$$
\# H \text {-orbits in } G \cdot \theta \cap \mathfrak{h}_{\chi}^{\perp}(\mathfrak{n}) \text {. }
$$

So we again consider the surjection

$$
G \cdot \varphi \cap \mathfrak{h}_{\chi}^{\perp}(\mathfrak{g}) \rightarrow G \cdot \theta \cap \mathfrak{h}_{\chi}^{\perp}(\mathfrak{n}) .
$$

This time it is not injective. Nevertheless, we can complete the argument if we show that the map sets up a bijection of $H$-orbits. Since the mapping is surjective and $H$-equivariant, it clearly maps $H$-orbits to $H$-orbits and is surjective on $H$-orbits. We need only show that distinct $H$-orbits in $G \cdot \varphi \cap \mathfrak{h}_{\chi}^{\perp}(\mathfrak{g})$ restrict to distinct $H$-orbits in $G \cdot \theta \cap \mathfrak{h}_{\chi}^{\perp}(\mathfrak{n})$. So suppose we have $\omega_{1}, \omega_{2} \in \Omega=$ $G \cdot \varphi$ and $h \in H$ such that

$$
\left.h \cdot \omega_{1}\right|_{\mathfrak{n}}=\left.\omega_{2}\right|_{\mathfrak{n}} .
$$

Set $\theta_{1}=\left.\omega_{1}\right|_{\mathrm{n}}$. The pair $\left(\omega_{1}, \theta_{1}\right)$ have the same properties as the pair $(\varphi, \theta)-$ namely those of case (a). Now there is $g \in G$ such that $\omega_{2}=g \cdot \omega_{1}$. Therefore

$$
h \cdot \theta_{1}=g \cdot \theta_{1} \text {. }
$$

Then $h^{-1} g \in G_{\theta_{1}}=N_{\theta_{1}}$. Now I claim that $N_{\theta_{1}} \cdot \omega_{1}=H_{\theta_{1}} \cdot \omega_{1}$. In fact $H_{\theta_{1}} \cdot \omega_{1} \subset N_{\theta_{1}} \cdot \omega_{1}=\omega_{1}+\mathbf{R} \alpha$. The only way the inclusion can be proper is if $H_{\theta_{1}} \cdot \omega_{1}=\omega_{1}$. But then $H_{\theta_{1}}=H_{\omega_{1}}$, which contradicts the equation $\operatorname{dim} H \cdot \theta_{1}=\operatorname{dim} H \cdot \omega_{1}-1$. Therefore, we must have $h_{1} \in H_{\theta_{1}}$ satisfying $h_{1} \cdot \omega_{1}=h^{-1} g \cdot \omega_{1}$. That is, $\omega_{2}=h h_{1} \cdot \omega_{1}$. Q.E.D.

Remarks 2.1. (i) Strictly speaking, in our proof of the equivalence of the measures in equation (A), our argument in case (a) showed equivalence of the $G$ push-forwards. To show the equivalence of the $H$-push-forwards requires the last idea in the above proof. Namely, the natural map

$$
\mathfrak{h}_{\chi}^{\perp}(\mathfrak{g}) / H \rightarrow \mathfrak{h}_{\chi}^{\perp}(\mathfrak{n}) / H
$$

is a bijection in case (a), because $H_{\theta} \cdot \varphi=\varphi+\mathbf{R} \alpha$.

(ii) In case (i) of the proof of Theorem 1.5 we are in the situation of an algebraic variety (e.g., $\left.G \cdot \varphi \cap \mathfrak{h}_{\chi}^{\perp}(\mathfrak{g})\right)$ acted upon by an algebraic group (e.g., $H$ ) for which all the orbits have the same dimension as the variety. This situation occurs again in $\S 4$. Whenever that is the case the number of orbits must be finite. In face the varieties that arise are generically manifolds (see the Appendix to this section), and the orbits are the connected components. That is more than we need to assert finite multiplicity.

(iii) Much of the current interest in the decomposition of the quasi-regular representation of $G$ on $L^{2}(G / H), H$ connected, comes from the theory of invariant differential operators (see, e.g., [15]). The Plancherel analysis of $G$ 
has been of enormous help in deriving solvability properties of left invariant differential operators on $G$. The feeling is that the same should be true of the differential operators obtained by passing to $G / H$-provided one can analyze the quasi-regular representation. Results like Theorem 1.5 are a first step in that direction. Nevertheless, although Theorem 1.5 gives both the spectrum and the multiplicity, it does not explicitly specify the exact intertwining operator which effects the decomposition. When $H=\{1\}$, the operator is the Fourier transform and that is critical to the success of the program [15]. Work continues by several people on the actual "Fourier" intertwining operator implicit in Theorem 1.5.

For future reference I wish to restate Theorem 1.5 in the case $\chi=1$.

Theorem 2.2. $\operatorname{Ind}_{H}^{G} 1=\int_{\mathfrak{h}^{\perp} / H}^{\oplus} \pi_{\varphi} d \mu_{G, H}(\varphi)$, where $\mu_{G, H}$ is the push-forward of Lebesgue measure under $\mathfrak{h}^{\perp} \rightarrow \mathfrak{h}^{\perp} / H$. The multiplicity in the above direct integral decomposition is $n_{\varphi}=\# H$-orbits in $G \cdot \varphi \cap \mathfrak{h}^{\perp}$.

\section{2a. APPENDIX}

The fact that in the equal dimension case, the varieties $G \cdot \varphi \cap \mathfrak{h}_{x}^{\perp}(\mathfrak{g})$ are (generically) manifolds is proved in [2], as usual, with the aid of layers and stratification. But it too can be derived purely by induction. Here are the main details.

Proposition 2.3. Suppose we are in condition (i) of either Corollary 1.3 or Lemma 1.4. Then generically on $\mathfrak{h}_{\chi}^{\perp}$, the algebraic varieties $G \cdot \varphi \cap \mathfrak{h}_{\chi}^{\perp}$ are smooth manifolds.

Proof. The proof is by induction on $\operatorname{dim} G / H$. If $\operatorname{dim} G / H=1$, the intersections are either points or lines (see Theorem 0.1 ) and there is nothing further to prove. Now assume $\operatorname{dim} G / H>1$, and the result is true in lower codimension. As usual, place $N$ between $H$ and $G, H \subset N \triangleleft G, \operatorname{dim} G / N=1$. By the induction assumption, generically on $\mathfrak{h}_{\chi}^{\perp}(\mathfrak{n})$ the varieties $N \cdot \theta \cap \mathfrak{h}_{\chi}^{\perp}(\mathfrak{n})$ are manifolds. We need to investigate $G \cdot \varphi \cap \mathfrak{h}_{\chi}^{\perp}(\mathfrak{g}), \varphi \in p_{\mathfrak{g}, \mathfrak{n}}^{-1}(\theta)$. We examine the two cases of Theorem 0.1. In case (b), $p(G \cdot \varphi)=N \cdot \theta$ and the manifold $G \cdot \varphi \cap \mathfrak{h}_{\chi}^{\perp}(\mathfrak{g})$ is algebraically isomorphic to $N \cdot \theta \cap \mathfrak{h}_{\chi}^{\perp}(\mathfrak{n})$. Nothing more need be said in that case. In case (a), however, the algebraic variety $G \cdot \varphi \cap \mathfrak{h}_{\chi}^{\perp}(\mathfrak{g})$ is one dimension larger than $N \cdot \theta \cap \mathfrak{h}_{\chi}^{\perp}(\mathfrak{n})$. But it is easy to see the manifold structure on the former. First of all, the variety $G \cdot \theta \cap \mathfrak{h}_{\chi}^{\perp}(\mathfrak{n})$ must have the same dimension as $N \cdot \theta \cap \mathfrak{h}_{\chi}^{\perp}(\mathfrak{n})$-both are $\operatorname{dim} H \cdot \theta$ (see proof of Theorem 1.5). In fact it is a finite disjoint union of "copies" of the manifold $N \cdot \theta \cap \mathfrak{h}_{\chi}^{\perp}(\mathfrak{n})$ and so is a manifold itself. Moreover, the mapping $G \cdot \varphi \cap \mathfrak{h}_{\chi}^{\perp}(\mathfrak{g}) \rightarrow G \cdot \theta \cap \mathfrak{h}_{\chi}^{\perp}(\mathfrak{n})$ is an algebraic submersion. But locally we can write $G \approx G / G_{\theta} \times G_{\theta}$. Moreover, in case (a) $G_{\theta}=N_{\theta}$ and $N_{\theta} \cdot \varphi=\varphi+\mathbf{R} \alpha$. Thus the submersion has fibers 
isomorphic to $\mathbf{R}$ and locally the map $g \cdot \varphi \rightarrow\left(g G_{\theta} \cdot \theta, t_{g}\right)$ corresponding to $g \sim\left(g G_{\theta}, g_{\theta}\right), \mathfrak{g} \cdot \varphi=\varphi+t_{g} \alpha$ gives smooth coordinates on $G \cdot \varphi \cap \mathfrak{h}_{\chi}^{\perp}(\mathfrak{g})$.

\section{Extension to $\operatorname{Ind}_{H}^{G} \nu$}

Corwin and Greenleaf are able to decompose the representation of $G$ induced by an arbitrary irreducible representation of the subgroup $H$, not just a character. It is possible to extend Theorem 1.5 to that situation as well. Let $G$ be simply connected nilpotent, $H$ a closed connected subgroup, $\nu \in \hat{H}$. We write $\mathscr{O}_{\nu}$ for the $H$-orbit in $\mathfrak{h}^{*}$ that corresponds to $\nu$ and $p=p_{\mathfrak{g}, \mathfrak{h}}$.

Theorem 3.1. We have

$$
\operatorname{Ind}_{H}^{G} \nu=\int_{p^{-1}\left(\mathscr{O}_{\nu}\right) / H}^{\oplus} \pi_{\varphi} d \mu_{G, H}^{\nu}(\varphi)
$$

where $\mu_{G, H}^{\nu}$ is the push-forward of the natural measure under

$$
p^{-1}\left(\mathscr{O}_{\nu}\right) \rightarrow p^{-1}\left(\mathscr{O}_{\nu}\right) / H
$$

What is the natural measure class on $p^{-1}\left(\mathscr{O}_{\nu}\right)$ ? It is the fiber measure class uniquely determined from the picture

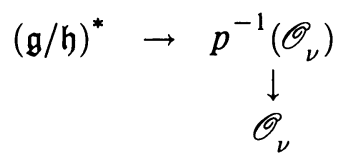

where the base has the canonical (Kirillov-Kostant) orbit measure and the fiber has Lebesgue measure.

It is possible to prove Theorem 3.1 by a case-by-case analysis analogous to the proof of Theorem 1.5 in $\S 2$. But in fact one can derive it from Theorem 1.5 using the fact that $\nu$ is induced from a character. This is actually done in $[2, \S 6]$, in truth without any real use of layers. I shall go through an outline of the argument, leaving out one detail that can be plucked off from $[2, \S 6]$.

Proof of Theorem 3.1. Every irreducible representation of a connected nilpotent Lie group is monomial, induced from a character. Choose $\psi \in \mathscr{O}_{\nu}$. Then one can find a real polarization $\mathfrak{k}$ of $\psi$ such that

$$
\nu=\operatorname{Ind}_{K}^{H} \chi,
$$

$\chi(\exp X)=e^{i \psi(X)}, X \in \mathfrak{k}$. By induction in stages we have

$$
\operatorname{Ind}_{H}^{G} \nu=\operatorname{Ind}_{H}^{G} \operatorname{Ind}_{K}^{H} \chi=\operatorname{Ind}_{K}^{G} \chi .
$$

Theorem 1.5 tells us how to decompose the latter. It is incumbent upon us to show that that is the same as the decomposition described in Theorem 3.1. That is, we need to show

$$
\int_{p^{-1}\left(\mathcal{O}_{\nu}\right) / H}^{\oplus} \pi_{\varphi} d \mu_{G, H}^{\nu}(\varphi) \simeq \int_{\mathfrak{l}_{\chi}^{\frac{1}{\chi}}(\mathbf{g}) / K}^{\oplus} \pi_{\varphi} d \mu_{G, K}^{\chi}(\varphi) .
$$


Well, first we observe that the spectra are the same. That is, the $G$-orbits in $\mathfrak{g}^{*}$ that meet $p^{-1}\left(\mathscr{O}_{\nu}\right)$ are exactly the same as the $G$-orbits that meet $\mathfrak{k}_{\chi}^{\perp}(\mathfrak{g})$. In fact, one inclusion is obvious: a $G$-orbit that meets the former must meet the latter. Conversely, suppose $\varphi \in \mathfrak{k}_{\chi}^{\perp}(\mathfrak{g})$. Then $\left.\psi\right|_{\mathfrak{k}}=\left.\varphi\right|_{\mathfrak{k}}$. But a necessary property of real polarizations in a nilpotent group is the equation $K \cdot \psi=\psi+\mathfrak{k}^{\perp}(\mathfrak{h})$. Therefore there is $k \in K$ such that $\left.k \cdot \varphi\right|_{\mathfrak{h}}=\psi$. In particular $k \cdot \varphi$ meets $p^{-1}\left(\mathscr{O}_{\nu}\right)$.

Next come the measures. First we observe that $H \cdot \mathfrak{k}_{\chi}^{\perp}(\mathfrak{g})=p^{-1}\left(\mathscr{O}_{\nu}\right)$. The former is also realized as a fiber space

$$
\begin{gathered}
\mathfrak{k}_{\chi}^{\perp}(\mathfrak{g}) \approx(\mathfrak{g} / \mathfrak{k})^{*} \rightarrow \quad H \cdot \mathfrak{k}_{\chi}^{\perp}(\mathfrak{g}) \\
\downarrow \\
H / K
\end{gathered}
$$

(the projection sends $\varphi \rightarrow h K$ if $\left.h \cdot \varphi\right|_{\mathfrak{k}}=-i d \chi$ ). But this space and the fiber space in the description of the natural measure are locally direct products. The former is locally $(\mathfrak{g} / \mathfrak{k})^{*} \times(\mathfrak{h} / \mathfrak{k})$; the latter is locally $\left.(\mathfrak{g} / \mathfrak{h})^{*} \times(\mathfrak{h} / \mathfrak{h})_{\psi}\right)$. Using the duality between $\mathfrak{h} / \mathfrak{k}$ and $\mathfrak{k} / \mathfrak{h}_{\psi}$, we see the spaces are locally isomorphic (actually globally) and carry the same Lebesgue measure class. The quotients $p^{-1}\left(\mathscr{O}_{\nu}\right) / H=H \cdot \mathfrak{k}_{\chi}^{\perp}(\mathfrak{g}) / H$ and $\mathfrak{k}_{\chi}^{\perp}(\mathfrak{g}) / K$ are identified-if $h \cdot \varphi_{1}=\varphi_{2},\left.\varphi_{i}\right|_{\mathfrak{t}}=$ $-i d \chi$, then $\left.\varphi_{2}\right|_{\mathfrak{h}}=\left.k \cdot \varphi_{1}\right|_{\mathfrak{h}}$ for some $k \in K$; which implies $k^{-1} h \in H_{\left(\varphi_{1} \mid \mathfrak{h}\right)} \subset K$, so $h \in K$. Therefore the quotient measure classes must be equivalent.

It remains to demonstrate equality of multiplicity. We know

$$
\int_{\mathfrak{k}_{x}^{\perp}(\mathfrak{g}) / K}^{\oplus} \pi_{\varphi}=\int_{G \cdot \mathfrak{k}_{x}^{\perp}(\mathfrak{g}) / G}^{\oplus} n_{\varphi, \chi} \pi_{\varphi}, \quad n_{\varphi, \chi}=\# K \text {-orbits on } G \cdot \varphi \cap \mathfrak{k}_{\chi}^{\perp}(\mathfrak{g}) .
$$

Next we claim

$$
\int_{p^{-1}\left(\mathscr{O}_{\nu}\right) / H}^{\oplus} \pi_{\varphi}=\int_{G \cdot p^{-1}\left(\mathscr{O}_{\nu}\right) / G}^{\oplus} n_{\varphi, \nu} \pi_{\varphi}, \quad n_{\varphi, \nu}=\# H \text {-orbits on } G \cdot \varphi \cap p^{-1}\left(\mathscr{O}_{\nu}\right) .
$$

In fact, this follows from our general principle in Lemma $1.2\left(p^{-1}\left(\mathscr{O}_{\nu}\right)\right.$ is not a linear subspace of $\mathfrak{g}^{*}$, but it does not matter). The proof is completed by the following:

Proposition 3.2. There is a bijection between $K$-orbits in $G \cdot \varphi \cap \mathfrak{k}_{\chi}^{\perp}(\mathfrak{g})$ and $H$ orbits in $G \cdot \varphi \cap p^{-1}\left(\mathscr{O}_{\nu}\right)$ given by the map

$$
K \cdot \varphi \rightarrow H \cdot \varphi .
$$

This is precisely Proposition 5 of [2]. The proof is not difficult-the ideas in the paragraph above on the equivalence of the measure classes are basically all that is required; see [2, pp. 52, 53]. And I emphasize, no layers are involved, just dimension counting and properties of polarizations in nilpotent groups. I also point out that in [2] it is shown why generically the orbit intersections $G \cdot \varphi \cap p^{-1}\left(\mathscr{O}_{\nu}\right)$ are smooth manifolds, not just algebraic varieties. The argument, like that of Proposition 3.2, does not depend on layers. 
This completes the proof of Theorem 3.1. But it should be mentioned that Corwin and Greenleaf do not state their result that way. Their formulation is rather more convoluted, like the statement of Theorem 1.1. So we need to do some work analogous to $\S \S 1.2-1.4$ to derive [2, Theorem 7] from Theorem 3.1. Indeed the analog of Lemma 1.2 is the statement that the multiplicity implicit in Theorem 3.1 is

$$
n_{\varphi, \nu}=\# H \text {-orbits on } G \cdot \varphi \cap p^{-1}\left(\mathscr{O}_{\nu}\right) .
$$

The analog of Corollary 1.3 follows immediately (using the nilpotence of $G$ and algebraicity of the adjoint action-see $\S 5$ ).

Corollary 3.3. Let $\varphi \in p^{-1}\left(\mathscr{O}_{\nu}\right)$ be generic.

(i) If $\operatorname{dim} H \cdot \varphi=\operatorname{dim} G \cdot \varphi \cap p^{-1}\left(\mathscr{O}_{\nu}\right)$, then $n_{\varphi, \nu}=\#$ conn. comp. of $G \cdot \varphi \cap p^{-1}\left(\mathscr{O}_{\nu}\right)$.

(ii) If $\operatorname{dim} H \cdot \varphi<\operatorname{dim} G \cdot \varphi \cap p^{-1}\left(\mathscr{O}_{\nu}\right)$, then $n_{\varphi, \nu}=+\infty$.

Finally, to obtain the Corwin-Greenleaf necessary and sufficient condition for 3.3(i) to hold, that is for finite multiplicity, we have the following:

Proposition 3.4. Generically on $p^{-1}\left(\mathscr{O}_{\nu}\right)$ we have

$$
\operatorname{dim} G \cdot \varphi \cap p^{-1}\left(\mathscr{O}_{\nu}\right)=\operatorname{dim} G \cdot \varphi-\operatorname{dim} H \cdot \varphi+\operatorname{dim} \mathscr{O}_{\nu} .
$$

Therefore either

(i) $\operatorname{dim} G \cdot \varphi=2 \operatorname{dim} H \cdot \varphi-\operatorname{dim} \mathscr{O}_{\nu}$, equivalently $n_{\varphi, \nu}$ is finite; or

(ii) $\operatorname{dim} G \cdot \varphi>2 \operatorname{dim} H \cdot \varphi-\operatorname{dim} \mathscr{O}_{\nu}$, equivalently $n_{\varphi, \nu}=+\infty$.

Proof. As before let $\psi \in \mathscr{O}_{\nu}, \psi=\left.\varphi\right|_{\mathfrak{h}}$. The first claim is that $\mathfrak{h}_{\psi} \cap \mathfrak{g}_{\varphi}=\mathfrak{h}_{\varphi}$. The inclusion $\subset$ is obvious. Conversely, let $X \in \mathfrak{h}_{\varphi}=\mathfrak{h} \cap \mathfrak{g}_{\varphi}$. Then $X \cdot \varphi=0$. Therefore $p(X \cdot \varphi)=X \cdot p(\varphi)=X \cdot \psi=0 \Rightarrow x \in \mathfrak{h}_{\psi}$. Now as usual it is enough (by looking at generic tangent spaces) to consider $\operatorname{dim} \mathfrak{g} \cdot \varphi \cap p^{-1}(\mathfrak{h} \cdot \psi)$. In fact we may compute (by footnote 2 and Proposition 1.7)

$$
\begin{aligned}
\operatorname{dim} \mathfrak{g} \cdot \varphi \cap p^{-1}(\mathfrak{h} \cdot \psi) & =\operatorname{dim}_{\mathfrak{g}_{\varphi}^{\perp} \cap p^{-1}\left(\mathfrak{h}_{\psi}^{\perp}(\mathfrak{h})\right)} \\
& =\operatorname{dim} \mathfrak{g}_{\varphi}^{\perp} \cap \mathfrak{h}_{\psi}^{\perp}(\mathfrak{g}) \\
& =\operatorname{dim}\left(\mathfrak{g}_{\varphi}+\mathfrak{h}_{\psi}\right)^{\perp} \\
& =\left(\operatorname{dim} \mathfrak{g}-\operatorname{dim} \mathfrak{g}_{\varphi}\right)-\left(\operatorname{dim} \mathfrak{h}_{\psi}-\operatorname{dim}\left(\mathfrak{h}_{\psi} \cap \mathfrak{g}_{\varphi}\right)\right) \\
& =\operatorname{dim} \mathfrak{g} \cdot \varphi-\left(\operatorname{dim} \mathfrak{h}_{\psi}-\operatorname{dim} \mathfrak{h}_{\varphi}\right) \\
& =\operatorname{dim} \mathfrak{g} \cdot \varphi-\left(\operatorname{dim} \mathfrak{h}-\operatorname{dim} \mathfrak{h}_{\varphi}\right)+\left(\operatorname{dim} \mathfrak{h}-\operatorname{dim} \mathfrak{h}_{\psi}\right) \\
& =\operatorname{dim} \mathfrak{g} \cdot \varphi-\operatorname{dim} \mathfrak{h} \cdot \varphi+\operatorname{dim} \mathfrak{O}_{\nu} . \text { Q.E.D. }
\end{aligned}
$$

We can now state the orbital spectral decomposition of $\operatorname{Ind}_{H}^{G} \nu$ in the manner in which it is presented in [2]. 
Theorem 3.5. $\operatorname{Ind}_{H}^{G} \nu=\int_{G \cdot p^{-1}\left(\mathscr{O}_{\nu}\right) / G}^{\oplus} n_{\varphi, \nu} \pi_{\varphi} d \tilde{\mu}_{G, H}^{\nu}(\varphi)$, where $G \cdot p^{-1}\left(\mathscr{O}_{\nu}\right) / G$ is the set of $G$-orbits that meet $p^{-1}\left(\mathscr{O}_{\nu}\right), \tilde{\mu}_{G, H}^{\nu}(\varphi)$ is the push-forward of the natural measure class under $p^{-1}\left(\mathscr{O}_{\nu}\right) \rightarrow G \cdot p^{-1}\left(\mathscr{O}_{\nu}\right) / G$, and $n_{\varphi, \nu}$ is given by: if generically on $p^{-1}\left(\mathscr{O}_{\nu}\right)$ we have

$$
\begin{aligned}
\operatorname{dim} G \cdot \varphi & >2 \operatorname{dim} H \cdot \varphi-\operatorname{dim} \mathscr{O}_{\nu} \text { then } n_{\varphi, \nu}=+\infty \\
\operatorname{dim} G \cdot \varphi & =2 \operatorname{dim} H \cdot \varphi-\operatorname{dim} \mathscr{O}_{\nu} \text { then } n_{\varphi, \nu} \\
& =\# H \text {-orbits on } G \cdot \varphi \cap p^{-1}\left(\mathscr{O}_{\nu}\right) \\
& =\# \text { conn. comp. of } G \cdot \varphi \cap p^{-1}\left(\mathscr{O}_{\nu}\right)<\infty
\end{aligned}
$$

In the future for nonnilpotent groups, I shall not try to generalize Theorem 1.1 or 3.5. As remarked in $\S 1$, they do not extend. It is the formulation in Theorems $1.5,2.2$, and 3.1 that may be generalized to exponential solvable groups and beyond.

Finally, let us note that $G \cdot p^{-1}\left(\mathscr{O}_{\nu}\right)=G \cdot p^{-1}(\psi)$ and that by arguments similar to several that occurred previously, we may show that $\tilde{\mu}_{G, H}^{\nu}$ is the pushforward of Lebesgue measure under $p^{-1}(\psi) \rightarrow G \cdot p^{-1}(\psi) / G$.

\section{RESTRICTIONS}

Now we take up the reciprocal problem of restricting irreducible representations of $G$ to a closed connected subgroup $H$. An orbital description of the spectra and multiplicities has been derived by Corwin and Greenleaf [4]. Moreover, their proof - again by layers and stratification-is substantially more complicated than that of the inducing problem. (This is due mainly to the lack of an analog for the step carried out in §3.) But in fact, as in the inducing situation, the argument can be dramatically simplified by reasoning by induction on $\operatorname{dim} G / H$ and utilizing the 4-case procedure of $\S 2$.

Let $G$ be simply connected nilpotent, $H$ a closed connected subgroup. Let $\mathscr{O} \subset \mathfrak{g}^{*}, \mathscr{U} \subset \mathfrak{h}^{*}$ be a $G$-orbit and $H$-orbit, respectively. Take $p=p_{\mathfrak{g}, \mathfrak{h}}$.

Definition 4.1. We set

$$
n_{\mathscr{O}, \mathscr{U}}=\# H \text {-orbits in } \mathscr{O} \cap p^{-1}(\mathscr{U}) .
$$

If $\varphi \in \mathscr{O}$ and $\psi \in \mathscr{U}$ we write $n_{\varphi, \psi}$ for $n_{\mathscr{O}, \mathscr{U}}$, where $\mathscr{O}=G \cdot \varphi, \mathscr{U}=H \cdot \psi$. Also if $\pi \in \hat{G}$ corresponds to $\mathscr{O}$ and $\nu \in \hat{H}$ corresponds to $\mathscr{U}$, we write $n_{\pi, \nu}=n_{\mathscr{G}, \mathscr{U}}$. On occasion we mix the notation, e.g., $n_{\varphi, \nu}$ as in $\S 3$.

The basic result is the following:

Theorem 4.2. Let $\varphi \in \mathfrak{g}^{*}, \pi=\pi_{\varphi} \in \hat{G}$. Then

$$
\left.\pi\right|_{H}=\int_{p(G \cdot \varphi) / H}^{\oplus} n_{\varphi, \psi} \nu_{\psi} d \lambda_{H, G}^{\pi}(\psi)
$$


where $\lambda_{H, G}^{\pi}$ is the push-forward of the canonical measure on $G \cdot \varphi$ under $G \cdot \varphi \rightarrow$ $p(G \cdot \varphi) / H$, and $n_{\varphi, \psi}$ is as specified in Definition 4.1 .

Proof. The argument is by induction on $\operatorname{dim} G / H$.

$\operatorname{dim} G / H=1 . H$ must be normal ( $G$ is nilpotent). In this case Theorem 4.2 is a special case of Theorem 6.2.

$\operatorname{dim} G / H>1$. The nilpotency of $G$ gives the existence of $N$, closed connected, $H \subset N \triangleleft G, \operatorname{dim} G / N=1$. By the induction hypothesis, the theorem is true for $N$ and $H$. Now since we are dealing with functionals, orbits, and representations on three different groups, let us be very systematic about notation:

$$
\begin{aligned}
& \varphi \in \mathfrak{g}^{*}, \theta \in \mathfrak{n}^{*}, \quad \psi \in \mathfrak{h}^{*}, \\
& \pi=\pi_{\varphi} \in \hat{G}, \quad \gamma=\gamma_{\theta} \in \hat{N}, \quad \nu=\nu_{\psi} \in \hat{H} .
\end{aligned}
$$

We may suppose these are all generic functionals. Indeed, for given $\varphi \in \mathfrak{g}^{*}$, generically on $p_{\mathfrak{g}, \mathfrak{h}}(G \cdot \varphi)$ it is true that $\varphi$ is generic for $\psi \in p_{\mathfrak{g}, \mathfrak{h}}(G \cdot \varphi)$; i.e., $\pi_{\varphi}$ is a generic representation in the induced representation $\operatorname{Ind}_{H}^{G} \nu_{\psi}$ (see Appendix to this section).

Now for the basic computation. By restriction in stages, Theorem 6.2, and the induction hypothesis, we have

$$
\begin{aligned}
\left.\pi_{\varphi}\right|_{H} & =\left.\left(\left.\pi_{\varphi}\right|_{N}\right)\right|_{H}=\left.\int_{p_{\mathfrak{\theta}, \mathrm{n}}(G \cdot \varphi) / N}^{\oplus} n_{\varphi, \theta} \gamma_{\theta}\right|_{H} d \lambda_{N, G}^{\varphi}(\theta) \\
& =\int_{p_{g, n}(G \cdot \varphi) / N}^{\oplus} \int_{p_{\mathrm{n}, \mathrm{h}}(N \cdot \theta) / H}^{\oplus} n_{\varphi, \theta} n_{\theta, \psi} \nu_{\psi} d \lambda_{H, N}^{\theta}(\psi) d \lambda_{N, G}^{\varphi}(\theta) \\
& =\int_{p_{\mathfrak{g}, \mathrm{h}}(G \cdot \varphi) / H}^{\oplus} n_{\varphi, \psi} \nu_{\psi} d \lambda_{H, G}^{\varphi}(\psi) .
\end{aligned}
$$

We must prove equation (B). For bookkeeping purposes, recall

$$
\begin{aligned}
& n_{\varphi, \psi}=\# H \text {-orbits in } p_{\mathfrak{g}, \mathfrak{h}}^{-1}\left(\mathscr{O}_{\nu}\right) \cap G \cdot \varphi, \\
& n_{\theta, \psi}=\# H \text {-orbits in } p_{\mathfrak{n}, \mathfrak{h}}^{-1}\left(\mathscr{O}_{\nu}\right) \cap N \cdot \theta, \\
& n_{\varphi, \theta}=\# N \text {-orbits in } p_{\mathfrak{g}, \mathfrak{n}}^{-1}(N \cdot \theta) \cap G \cdot \varphi .
\end{aligned}
$$

In fact the numbers $n_{\varphi, \theta}$ are identically 1 . To see that, let us specify our two dichotomous situations again: Either

(i) $\operatorname{dim} G \cdot \varphi=2 \operatorname{dim} H \cdot \varphi-\operatorname{dim} H \cdot \psi$ or

(ii) $\operatorname{dim} G \cdot \varphi>2 \operatorname{dim} H \cdot \varphi-\operatorname{dim} H \cdot \psi$

and either

(a) $\operatorname{Ind}_{N}^{G} \nu_{\theta}$ is irreducible or

(b) $\operatorname{Ind}_{N}^{G} \nu_{\theta}=\int^{\oplus} \pi_{\varphi+t \alpha} d t$.

Referring back to Theorem 0.1 for the restriction aspect of (a) and (b) we know that in case (a)

$\left.\pi_{\varphi}\right|_{N}=\int \gamma_{\theta_{s}} d s, \quad$ multiplicity free and $p_{\mathfrak{g}, \mathfrak{n}}^{-1}\left(N \cdot \theta_{s}\right)=N s \cdot \varphi$ has one $N$-orbit ; 
whereas in case (b)

$$
\left.\pi_{\varphi}\right|_{N}=\gamma_{\theta} \text { and } p^{-1}(N \cdot \theta) \cap G \cdot \varphi=N \cdot \varphi \text { also has one } N \text {-orbit . }
$$

(The latter is because $\left.g \cdot \varphi\right|_{\mathfrak{n}}=n \cdot \theta \Rightarrow n^{-1} g \in G_{\theta}=G_{\varphi} \Rightarrow g \cdot \varphi=n \cdot \varphi$.) Thus the numbers $n_{\varphi, \theta}$ are generically 1 .

We first prove that the representations on the two sides of equation (B) are quasi-equivalent by showing that the spectra are the same and the measures are equivalent. Then we establish equal multiplicity by looking at the four cases separately. Equal spectra is almost obvious. On the right side, the spectrum consists of the irreducible representations of $H$ whose orbits lie in $p_{\mathfrak{g}, \mathfrak{h}}(G \cdot \varphi)$. On the left, it is the representations whose orbits lie in $p_{\mathfrak{n}, \mathfrak{h}}\left(N \cdot p_{\mathfrak{g}, \mathfrak{n}}(G \cdot \varphi)\right)=$ $p_{\mathfrak{n}, \mathfrak{h}}\left(p_{\mathfrak{g}, \mathfrak{n}}(G \cdot \varphi)\right)=p_{\mathfrak{g}, \mathfrak{h}}(G \cdot \varphi)$. The measures are also the same. In case (b) this is absolutely obvious since $p_{\mathfrak{g}, \mathfrak{n}}(G \cdot \varphi)=N \cdot \theta$ and the measures on either side are actually identical. In case (a) we have $p_{\mathfrak{g}, \mathfrak{n}}(G \cdot \varphi)=\bigcup_{s} N \cdot \theta_{s}$ and a fiber space

$$
\begin{array}{ccc}
N \cdot \theta_{s} \rightarrow p_{\mathfrak{g}, \mathfrak{n}}(G \cdot \varphi) \\
\downarrow \\
G / N .
\end{array}
$$

Moreover, the push-forward of the canonical measure on $G \cdot \varphi$ is the natural fiber measure (i.e., Lebesgue measure on the base and the canonical measure on the fiber). The entire picture is $H$-equivariant, so it factors to a fiber space

$$
\begin{gathered}
p_{\mathfrak{n}, \mathfrak{h}}\left(N \cdot \theta_{s}\right) / H \rightarrow p_{\mathfrak{g}, \mathfrak{h}}(G \cdot \varphi) / H \\
\downarrow \\
G / N
\end{gathered}
$$

and the measure classes on the two sides of equation (B) are seen to be equal.

Now we turn to our case-by-case verification of equal multiplicity. We first consider case (b). In that situation, the outer integration on the left side of (B) is a point evaluation, and we must prove $n_{\varphi, \psi}=n_{\theta, \psi}$ for generic $\psi$.

(iib) Here we have for generic $\psi, \operatorname{dim} G \cdot \varphi>2 \operatorname{dim} H \cdot \varphi-\operatorname{dim} H \cdot \psi$. By Proposition 3.4 we have $n_{\varphi, \psi}=+\infty$. The relevant facts in case (b) are $\left.\pi_{\varphi}\right|_{N}=\gamma_{\theta}$ and $p_{\mathfrak{g}, \mathfrak{n}}(G \cdot \varphi)=N \cdot \theta$. We also have $\operatorname{dim} \mathfrak{g} \cdot \varphi=\operatorname{dim} \mathfrak{n} \cdot \theta$ and $\mathfrak{g}_{\varphi}=\mathfrak{g}_{\theta}$, so that $\mathfrak{h}_{\varphi}=\mathfrak{h}_{\theta}$. Therefore

$$
\begin{aligned}
\operatorname{dim} \mathfrak{n} \cdot \theta & =\operatorname{dim} \mathfrak{g} \cdot \varphi>2 \operatorname{dim} \mathfrak{h} \cdot \varphi-\operatorname{dim} \mathfrak{h} \cdot \psi \\
& =2 \operatorname{dim} \mathfrak{h} \cdot \theta-\operatorname{dim} \mathfrak{h} \cdot \psi .
\end{aligned}
$$

Hence, Proposition 3.4 gives $n_{\theta, \psi}=+\infty$.

(ib) We continue with $\left.\pi_{\varphi}\right|_{N}=\gamma_{\theta}$, but this time $\operatorname{dim} G \cdot \varphi=2 \operatorname{dim} H \cdot \varphi-$ $\operatorname{dim} H \cdot \psi$. The exact same argument as in (iib) shows that

$$
\operatorname{dim} N \cdot \theta=2 \operatorname{dim} H \cdot \theta-\operatorname{dim} H \cdot \psi .
$$

Therefore in this case both $n_{\varphi, \psi}$ and $n_{\theta, \psi}$ are finite. Why are they equal? First of all, the canonical projection

$$
G \cdot \varphi \cap p_{\mathfrak{g}, \mathfrak{h}}^{-1}\left(\mathscr{O}_{\nu}\right) \rightarrow G \cdot \theta \cap p_{\mathfrak{n}, \mathfrak{h}}^{-1}\left(\mathscr{O}_{\nu}\right)
$$


is a bijection. It is obviously surjective. It is injective because if $\left.g_{1} \cdot \varphi\right|_{\mathfrak{n}}=$ $\left.g_{2} \cdot \varphi\right|_{\mathfrak{n}}$, then $g_{1} \cdot \varphi=g_{2} \cdot \varphi$ (since $G_{\theta}=G_{\varphi}$-see case (b) in $\left.\S 3\right)$. Next I claim that

$$
G \cdot \theta \cap p_{n, \mathfrak{h}}^{-1}\left(\mathscr{O}_{\nu}\right)=N \cdot \theta \cap p_{\mathrm{n}, \mathfrak{h}}^{-1}\left(\mathscr{O}_{\nu}\right) .
$$

The inclusion $\supset$ is obvious, and the reverse inclusion follows from $G=N G_{\theta}$. Hence $n_{\varphi, \psi}=\# H$-orbits on $G \cdot \varphi \cap p_{\mathfrak{g}, \mathfrak{h}}^{-1}\left(\mathscr{O}_{\nu}\right)$ must be the same as $n_{\theta, \psi}=\# H$ orbits on $N \cdot \theta \cap p_{\mathrm{n}, \mathfrak{h}}^{-1}\left(\mathscr{O}_{\nu}\right)$.

The argument in case (a) is considerably more subtle. We restate equation (B) in that case

$$
\int^{\oplus} \int_{p\left(N \cdot \theta_{s}\right) / H}^{\oplus} n_{\theta_{s}, \psi} \nu_{\psi} d \lambda_{H, N}^{\theta_{s}}(\psi) d s=\int_{p\left(G^{*} \varphi\right) / H}^{\oplus} n_{\varphi, \psi} \nu_{\psi} d \lambda_{H, G}^{\varphi}(\psi) .
$$

(iia) Now we have $\left.\pi_{\varphi}\right|_{N}=\int^{\oplus} \gamma_{\theta_{s}} d s$, and in the first instance we assume unequal dimension, i.e.,

$$
\operatorname{dim} G \cdot \varphi>2 \operatorname{dim} H \cdot \varphi-\operatorname{dim} H \cdot \psi .
$$

We know that $n_{\varphi, \psi}$ is infinite. We show that $n_{\theta_{s}, \psi}=\# H$-orbits on $N \cdot \theta_{s} \cap$ $p_{\mathrm{n}, \mathfrak{h}}^{-1}\left(\mathscr{O}_{\nu}\right)$ is also infinite for a.a.s. We know, by Theorem 0.1 , that

$$
\operatorname{dim} N \cdot \theta=\operatorname{dim} G \cdot \varphi-2 \text {. }
$$

Next we reason as in case (a) of $\S \S 2$ and 3 (more precisely $\S 2$ (iia) and $\S 3$ proof of Proposition 3.4):

$$
\begin{aligned}
\operatorname{dim} \mathfrak{g} \cdot \theta \cap p_{\mathfrak{n}, \mathfrak{h}}^{-1}(\mathfrak{h} \cdot \psi) & =\operatorname{dim} \mathfrak{n}_{\varphi}^{\perp} \cap p_{\mathfrak{n}, \mathfrak{h}}^{-1}\left(\mathfrak{h}_{\psi}^{\perp}(\mathfrak{h})\right) \\
& =\operatorname{dim} \mathfrak{n}_{\varphi}^{\perp} \cap \mathfrak{h}_{\psi}^{\perp}(\mathfrak{n}) \\
& =\operatorname{dim}\left(\mathfrak{n}_{\varphi}+\mathfrak{h}_{\psi}\right)^{\perp} \\
& =\left(\operatorname{dim} \mathfrak{n}-\operatorname{dim} \mathfrak{n}_{\varphi}\right)-\left(\operatorname{dim} \mathfrak{h}_{\psi}-\operatorname{dim} \mathfrak{h}_{\varphi}\right) \\
& =\operatorname{dim} \mathfrak{g} \cdot \theta-\operatorname{dim} \mathfrak{h} \cdot \varphi+\operatorname{dim} \mathscr{O}_{\nu} .
\end{aligned}
$$

We know $\operatorname{dim} \mathfrak{g} \cdot \theta=\operatorname{dim} \mathfrak{g} \cdot \varphi-1$. Since both sides of the inequality

$$
\operatorname{dim} \mathfrak{g} \cdot \varphi>2 \operatorname{dim} \mathfrak{h} \cdot \varphi-\operatorname{dim} \mathscr{O}_{\nu}
$$

are even, we have

$$
\operatorname{dim} \mathfrak{g} \cdot \theta>2 \operatorname{dim} \mathfrak{h} \cdot \varphi-\operatorname{dim} \mathfrak{h} \cdot \psi
$$

Hence

$$
\operatorname{dim} G \cdot \theta \cap p_{\mathfrak{n}, \mathfrak{h}}^{-1}\left(\mathscr{O}_{\nu}\right)>\operatorname{dim} H \cdot \varphi \geq \operatorname{dim} H \cdot \theta .
$$

At this point we cannot simply deduce $n_{\theta, \psi}=+\infty$ because $\operatorname{dim} N \cdot \theta \cap p_{\mathfrak{n}, \mathfrak{h}}^{-1}\left(\mathscr{O}_{\nu}\right)$ may be one less than that of $G \cdot \theta \cap p_{\mathfrak{n}, \mathfrak{h}}^{-1}\left(\mathscr{O}_{\nu}\right)$. (In $\S 2$ the dimension inequality with $G \cdot \theta$ was enough to deduce infinite multiplicity.) But we can reason as follows. We have

$$
G \cdot \theta=\bigcup_{s} N s \cdot \theta=\bigcup_{s} N \cdot \theta_{s} .
$$


Therefore $G \cdot \theta \cap p^{-1}\left(\mathscr{O}_{\nu}\right)=\bigcup_{s} N \cdot \theta_{s} \cap p^{-1}\left(\mathscr{O}_{\nu}\right)$. As always $\psi \in p(G \cdot \varphi)$ is generic. Suppose there is a set of positive measure $X$ such that

$$
\operatorname{dim} N \cdot \theta_{s} \cap p^{-1}\left(\mathscr{O}_{\nu}\right)=\operatorname{dim} G \cdot \theta \cap p^{-1}\left(\mathscr{O}_{\nu}\right), \quad \forall s \in X .
$$

Then $n_{\theta_{s}, \psi}=+\infty, s \in X$, and there is nothing further to prove in this case. Otherwise

$$
\operatorname{dim} N \cdot \theta_{s} \cap p^{-1}\left(\mathscr{O}_{\nu}\right)<\operatorname{dim} G \cdot \theta \cap p^{-1}\left(\mathscr{O}_{\nu}\right) \text { a.a.s } \in \mathbf{R} .
$$

It is possible then that $\operatorname{dim} H \cdot \theta_{s}=\operatorname{dim} N \cdot \theta_{s} \cap p^{-1}\left(\mathscr{O}_{\nu}\right)$ a.a.s $\in \mathbf{R}$, and then $n_{\theta_{s}, \psi}<\infty$, a.a.s. But note that in such an eventuality, we must have a set of positive measure $Y$ such that $N \cdot \theta_{s} \cap p^{-1}\left(\mathscr{O}_{\nu}\right) \neq \varnothing$. Then for all $s \in Y$, we have $p\left(N \cdot \theta_{s}\right) \cap p p^{-1}\left(\mathscr{O}_{\nu}\right) \neq \varnothing$, which says that $\nu$ occurs with infinite multiplicity in

$$
\left.\int_{s \in Y}^{\oplus} \int_{p\left(N \cdot \theta_{s}\right) / H}^{\oplus} \gamma_{\theta_{s}}\right|_{H} .
$$

Either way we get infinite multiplicity on both sides of equation (B).

We come at last to the trickiest case.

(ia) This time $\left.\pi_{\varphi}\right|_{N}=\int^{\oplus} \gamma_{\theta_{s}} d s$ and we are in the equal dimension situation, $\operatorname{dim} G \cdot \varphi=2 \operatorname{dim} H \cdot \varphi-\operatorname{dim} \mathscr{O}_{\nu}$. Then $n_{\varphi, \psi}$ is finite (generically). We must show that it agrees with the multiplicity on the left side of equation (B). First we show that (generically) the multiplicities $n_{\theta_{s}, \psi}$ are finite. Indeed

$$
\begin{aligned}
2 \operatorname{dim} H \cdot \theta & \leq \operatorname{dim} N \cdot \theta+\operatorname{dim} \mathscr{O}_{\nu} \\
& =\operatorname{dim} G \cdot \varphi-2+\operatorname{dim} \mathscr{O}_{\nu} \\
& =2 \operatorname{dim} H \cdot \varphi-2 .
\end{aligned}
$$

Hence

$$
\operatorname{dim} H \cdot \theta=\operatorname{dim} H \cdot \varphi-1 .
$$

This, together with the equations

$$
\operatorname{dim} N \cdot \theta=\operatorname{dim} G \cdot \varphi-2 \text { and } \operatorname{dim} G \cdot \varphi=2 \operatorname{dim} H \cdot \varphi-\operatorname{dim} \mathscr{O}_{\nu},
$$

implies that

$$
\operatorname{dim} N \cdot \theta=\operatorname{dim} H \cdot \theta-\operatorname{dim} \mathscr{O}_{\nu} .
$$

Hence

$$
\operatorname{dim} H \cdot \theta=\operatorname{dim} N \cdot \theta \cap p^{-1}\left(\mathscr{O}_{\nu}\right) .
$$

Allowing $s$ to vary now, we see that either

$$
\operatorname{dim} H \cdot \theta_{s}=\operatorname{dim} N \cdot \theta_{s} \cap p^{-1}\left(\mathscr{O}_{\nu}\right) \text { or } N \cdot \theta_{s} \cap p^{-1}\left(\mathscr{O}_{\nu}\right)=\varnothing .
$$

In particular, the multiplicities $n_{\theta_{s}, \psi}$ are finite (see Remark 2.1(ii)).

Now it is not true that $n_{\varphi, \psi}=n_{\theta, \psi}$-it is more subtle than that. Well, exactly as in (ia) of $\S 2$ we have a surjection

$$
G \cdot \varphi \cap p_{\mathfrak{g}, \mathfrak{h}}^{-1}\left(\mathscr{O}_{\nu}\right) \rightarrow G \cdot \theta \cap p_{n, \mathfrak{h}}^{-1}\left(\mathscr{O}_{\nu}\right)
$$


which sets up a bijection of $H$-orbits. Once again we have

$$
G \cdot \theta \cap p^{-1}\left(\mathscr{O}_{\nu}\right) \rightarrow \bigcup_{s} N \cdot \theta_{s} \cap p^{-1}\left(\mathscr{O}_{\nu}\right)
$$

By the above remarks, we must have that all but a finite number of the intersections in the above union are empty. That is, there is a finite number $s_{1}, \ldots, s_{r}$ (which will depend on $\psi$ in general) such that

$$
G \cdot \theta \cap p^{-1}\left(\mathscr{O}_{\nu}\right)=\bigcup_{i=1}^{r} N \cdot \theta_{s_{i}} \cap p^{-1}\left(\mathscr{O}_{\nu}\right),
$$

a disjoint union. Moreover,

$$
\begin{aligned}
n_{\varphi, \psi} & =\# H \text {-orbits in } G \cdot \theta \cap p^{-1}\left(\mathscr{O}_{\nu}\right) \\
& =\sum_{i=1}^{r} n_{\theta_{s_{i}}, \psi} .
\end{aligned}
$$

But the latter is precisely the multiplicity in the direct integral of the left side of equation (B). This proves equality of multiplicity in equation (B) and completes our proof of Theorem 4.2.

\section{4a. AppendiX}

We show here that if $\varphi \in \mathfrak{g}^{*}$ and $\psi \in p(G \cdot \varphi)$ is generic, then $\varphi$ is generic for $p^{-1}(H \cdot \psi)$, i.e., $\pi_{\varphi}$ is a generic representation in $\operatorname{Ind}_{H}^{G} \nu_{\psi}$. Our proof is, as expected, by induction on $\operatorname{dim} G / H$.

$\operatorname{dim} G / H=1$. Then $H$ is normal and the result follows immediately from Theorem 0.1 .

$\operatorname{dim} G / H>1$. As usual, we insert $N$ between $H$ and $G, H \subset N \triangleleft G$, $\operatorname{dim} G / N=1$. By the induction assumption, the result is true for the pair $H \subset N$. Let $\varphi \in \mathfrak{g}^{*}, \psi \in p_{\mathfrak{g}, \mathfrak{h}}(G \cdot \varphi)$. We distinguish between cases (a) and (b) of Theorem 0.1 .

Case (b). Then $p_{\mathfrak{g}, \mathfrak{n}}(G \cdot \varphi)=N \cdot \theta$, so $\psi \in p_{\mathfrak{n}, \mathfrak{h}}(N \cdot \theta)$ is generic. By induction, $\theta$ is generic for $p_{\mathfrak{n}, \mathfrak{h}}^{-1}(H \cdot \psi)$. But $N \cdot \theta=N \cdot \theta_{s}$ and so all the functionals $\theta_{s}$ are generic. Finally, since

$$
\operatorname{Ind}_{H}^{G} \gamma_{\theta}=\int^{\oplus} \pi_{\varphi+t \alpha} d t
$$

it is clear that $\varphi$ is generic for $p_{\mathfrak{g}, \mathfrak{h}}^{-1}(H \cdot \psi)$.

Case (a). Then $p_{\mathfrak{g}, \mathfrak{h}}(G \cdot \varphi)=\bigcup_{s} p_{\mathfrak{n}, \mathfrak{h}}\left(N \cdot \theta_{s}\right)$. Then for some $s, \psi \in$ $p_{\mathfrak{n}, \mathfrak{h}}\left(N \cdot \theta_{s}\right)$ and is generic there. Once again the induction argument applies, giving $\theta_{s}$ generic for $p_{\mathfrak{n}, \mathfrak{h}}^{-1}(H \cdot \psi)$. Since $\operatorname{Ind}_{H}^{G} \gamma_{\theta_{s}}=\pi_{\varphi}, \varphi$ is generic for $p_{\mathfrak{g}, \mathfrak{h}}^{-1}(H \cdot \psi)$. Q.E.D. 


\section{MORE REFINED RESULTS ON MULTIPLICITY}

Substantial emphasis is placed in [2] on the fact that the stratification by layers method enables one to deduce that in the decomposition of $\operatorname{Ind}_{H}^{G} \nu$ one has either uniform infinite multiplicity or bounded finite multiplicity. But in fact layers are not needed-that result (and more) is an elementary consequence of some standard facts from real algebraic geometry.

Proposition 5.1. For either the induced representation $\operatorname{Ind}_{H}^{G} \nu$ or the restriction $\left.\pi\right|_{H}$, the spectral multiplicity function satisfies the condition: either it is identically $+\infty$ or else it is finite and bounded.

Proof. First of all, by induction in stages, it is enough to prove the first half of the proposition for the case that $\nu$ is a character $\chi$. According to Theorem 1.5, Lemma 1.4, and Corollary 1.3 , either $\operatorname{Ind}_{H}^{G} \chi$ is of uniform infinite multiplicity or generically on $h_{\chi}^{\perp}$ the multiplicity is given by

$$
\text { \#conn.comp. of } G \cdot \varphi \cap \mathfrak{h}_{\chi}^{\perp} \text {. }
$$

Thus we must show the latter is finite and uniformly bounded over the orbits that intersect $\mathfrak{h}_{\chi}^{\perp}$. But the coadjoint action is defined by polynomials; i.e., there exist coordinates on $\mathfrak{g}^{*}$ and $n=\operatorname{dim} \mathfrak{g}$ polynomial functions $p_{j}(g, \varphi), g \in G$, $\varphi \in \mathfrak{g}^{*}$, such that

$$
g \cdot \varphi=\left(p_{1}(g, \varphi), \ldots, p_{n}(g, \varphi)\right) .
$$

Furthermore

$$
G \cdot \varphi \cap \mathfrak{h}_{\chi}^{\perp}=\left\{g \cdot \varphi: \sum_{j=1}^{n} a_{i j} p_{j}(g, \varphi)=\lambda_{i}, i=1, \ldots, r=\operatorname{dim} \mathfrak{h}\right\},
$$

for certain real numbers $a_{i j}, \lambda_{i}$, which depend only on $\mathfrak{h}$ and $\chi$. Writing

$$
\mathscr{V}_{a, \lambda, \varphi}=\bigcap_{i=1}^{r}\left\{g \cdot \varphi: \sum_{j=1}^{n} a_{i j} p_{j}(g, \varphi)=\lambda_{i}\right\},
$$

we see from Theorem 1.5 that (in the equal dimension case)

$$
n_{\varphi, \chi}=n_{a, \lambda, \varphi}=\# \text { conn.comp. of } \mathscr{V}_{a, \lambda, \varphi} .
$$

By Whitney's theorem [17], $n_{\varphi, \chi}<\infty$. The assertion of the proposition is that

$$
\sup _{\varphi} n_{a, \lambda, \varphi}<\infty .
$$

But in fact more is true. By [17], we have

$$
\sup _{a, \lambda, \varphi} n_{a, \lambda, \varphi}<\infty \text {. }
$$

An upper bound on the number of components depends only on the degree of the polynomials $p_{j}$ and the number of variables $n=\operatorname{dim} \mathfrak{g}$, i.e., only on the 
structure of $G$ itself. This proves the assertion about the multiplicity of the induced representation.

In fact, the inequality (1) implies the assertion for the restricted representation as well. Indeed, for $\varphi \in \mathfrak{g}^{*}$, the multiplicities in $\left.\pi_{\varphi}\right|_{H}$ are given (by Theorem 4.1) for $\nu \in \hat{H}$ by $n_{\varphi, \nu}=\# H$-orbits in $G \cdot \varphi \cap \mathfrak{h}^{\perp}\left(\mathscr{O}_{\nu}\right)$. If $n_{\varphi, \nu}>0$, we know there exists $\mathfrak{k} \subset \mathfrak{h}$ and $\psi \in \mathfrak{k}^{*}, \psi[\mathfrak{k}, \mathfrak{k}]=0$, such that $n_{\varphi, \nu}=n_{\varphi, \chi}=\# K$ orbits in $G \cdot \varphi \cap \mathfrak{k}_{\chi}^{\perp}$. Since the bound in (1) is independent of $a$ and $\lambda$, i.e., of $\mathfrak{h}$ or $\mathfrak{k}$ or $\psi$, it must be that the multipliities $n_{\varphi, \nu}$ are bounded as a function of $\nu$. (Note we are using that on $p(G \cdot \varphi)$ the generic $\operatorname{dim} G \cdot \varphi \cap p^{-1}(\psi)$ must be constant, so that we have either uniform infinite multiplicity or finite multiplicity given by the number of components in the intersection.)

Remark 5.2. Corwin and Greenleaf have obtained another very interesting multiplicity result in [3] - namely that in the finite multiplicity case, the parity of the multiplicity function is constant.

\section{PART II. NONNILPOTENT GROUPS}

Our ultimate goal is to generalize the results of Part I as far as possible to nonnilpotent groups. The guiding philosophy is the orbit method. It suggests that our main results (Theorems $1.5,2.2,3.1,4.2$ ) should generalize unchanged to exponential solvable groups and in modified-but quite similar-form to general Lie groups. We shall be concerned with both types of generalizations in the future. Thus the three sections of Part II should be viewed as preamble to this effort. $\S \S 6$ and 7 are critical to the exponential solvable case (although $\S 7$ has broader application). $\S 8$ contains a variety of nonnilpotent examples.

\section{NORMAL COSET SPACES}

In this section we shall give orbital descriptions of both induced and restricted representations in the case that the groups are exponential solvable and the subgroup is normal. The results of this section do not depend on anything from Part I. They are a blend of the orbit picture for exponential solvable groups with the Mackey machine (and some material from [10]).

Let $G$ be simply connected exponential solvable, $N \triangleleft G$ a closed connected normal subgroup. We do not assume $\operatorname{dim} G / N=1$ here.

Theorem 6.1. Let $\theta \in \mathfrak{n}^{*}, \gamma=\gamma_{\theta} \in \hat{N}$ the corresponding representation, $p=$ $p_{\mathfrak{g}, \mathfrak{n}}$. Then

$$
\operatorname{Ind}_{N}^{G} \gamma_{\theta}=\int_{p^{-1}(N \cdot \theta) / N}^{\oplus} \pi_{\varphi} d \mu_{G, N}^{\gamma}(\varphi)
$$

where $\mu_{G, H}^{\gamma}$ is the push-forward of the canonical measure on $p^{-1}(N \cdot \theta)$ under $p^{-1}(N \cdot \theta) \rightarrow p^{-1}(N \cdot \theta) / N$. Moreover, the multiplicity in the direct integral is identically 1 or $+\infty$, according as $\operatorname{dim} N \cdot \varphi=\operatorname{dim}\left(G \cdot \varphi \cap p^{-1}(N \cdot \theta)\right)$ or not, 
for generic $\varphi \in p^{-1}(N \cdot \theta) ;$ equivalently $\operatorname{dim} G \cdot \varphi=2 \operatorname{dim} N \cdot \varphi-\operatorname{dim} N \cdot \theta$ or not. In either case, the multiplicity $n_{\varphi, \theta}$ of $\pi_{\varphi}$ in $\operatorname{Ind}_{H}^{G} \gamma_{\theta}$ is \#N-orbits in $G \cdot \varphi \cap p^{-1}(N \cdot \theta)$.

Note that Theorems 3.1, 3.5 specialize to the first statement of Theorem 6.1 when $H$ is normal. The new property of uniform multiplicity 1 or $\infty$ is an added feature of the normal situation.

Proof. We begin with a quick presentation of the Mackey machine and its interactions with the orbit method on exponential solvable Lie groups. Since $G$ is exponential solvable, the closed subgroups $G_{\theta}$ and $G_{\gamma}=G_{\theta} N$ are connected. Let $\tilde{\gamma}$ be an extension of $\gamma$ to $G_{\gamma}$ satisfying $\tilde{\gamma}(g) \gamma\left(g^{-1} n g\right)=\gamma(n) \tilde{\gamma}(g), n \in N$, $g \in G_{\gamma} . \tilde{\gamma}$ may be a projective representation-as such it determines a multiplier or cocycle $\omega_{\gamma}$ on $G_{\gamma}$ which drops down to $G_{\gamma} / N$. $\tilde{\gamma}$ is not unique, but the class of $\omega_{\gamma}$ is. The multiplier $\omega_{\gamma}$ is exactly the obstruction to extending $\gamma$ to an ordinary representation of $G_{\gamma}$, and that is the same as the obstruction to extending $\chi_{\theta}=e^{i \theta}$ from $N_{\theta}$ to a character of $G_{\theta}$ [5].

Now it is proved in [10] that

$$
\operatorname{Ind}_{N}^{G} \gamma_{\theta}=\int_{\left(G_{i / N}^{\ominus} / N\right)^{\omega_{\gamma}}}^{\oplus}(\operatorname{dim} \sigma) \pi_{\gamma, \sigma} d \mu_{\gamma}(\sigma) .
$$

The meanings of the symbols are as follows: $\left(G_{\gamma} / N\right)^{\bar{\omega}_{y}}$ denotes the $\bar{\omega}_{\gamma}$-represen tations of $G_{\gamma} / N, \pi_{\gamma, \sigma}=\operatorname{Ind}_{G_{\gamma}}^{G} \sigma \otimes \tilde{\gamma}$ are the irreducible representations of $G$ specified by the Mackey machine, and $\mu_{\gamma}$ is the projective Plancherel measure on $\left(G_{\gamma} / N\right)^{\bar{\omega}_{y}}$. The group $G_{\gamma} / N$ is also exponential solvable, and therefore it must be that the multiplicity

$$
\operatorname{dim} \sigma= \begin{cases}1, & G_{\gamma} / N \text { abelian and } \omega_{\gamma} \text { trivial }, \\ \infty, & \text { otherwise }\end{cases}
$$

Now we must show that the direct integrals in equations (2) and (3) are the same. This requires-as in the proofs of equations (A) and (B) in Part I-that we show the spectra are the same, the measures are equivalent, and the multiplicities are identical. That the spectra are the same is an easy consequence of the orbit method. The linear functionals in $\mathfrak{g}^{*}$ that give rise to representations in $\hat{G}$ that lie over $\gamma=\gamma_{\theta}$ in the Mackey machine are exactly those in $G \cdot p^{-1}(N \cdot \theta)=G \cdot p^{-1}(\theta)[6,7]$. But the mapping

$$
p^{-1}(N \cdot \theta) \rightarrow G \cdot p^{-1}(N \cdot \theta) / G=G \cdot p^{-1}(\theta) / G
$$

is surjective. So the spectra of (2) and (3) are the same.

Next we examine the multiplicities. Consider the orbits $\Omega$ in $\mathfrak{g}^{*}$ which satisfy $\Omega \cap p^{-1}(N \cdot \theta) \neq \varnothing$. Let $\varphi \in \Omega,\left.\varphi\right|_{\mathfrak{n}}=\theta$. Then if $\left.g \cdot \varphi\right|_{\mathfrak{n}} \in N \cdot \theta$ we must have $n \in N$ such that $n^{-1} g \in G_{\theta}$. That is, $g \in G_{\theta} N$. Conversely, if 
$g \in G_{\theta} N$, then $\left.g \cdot \varphi\right|_{\mathfrak{n}} \in N \cdot \theta$. Hence:

if $\pi \in \hat{G}$ and $\Omega_{\pi}$ is the corresponding orbit, then either $\Omega_{\pi} \cap$ $p^{-1}(N \cdot \theta)=\varnothing$ or $\Omega_{\pi} \cap p^{-1}(N \cdot \theta)=G_{\theta} N \cdot \varphi$, for some $\varphi \in$ $\Omega_{\pi} \cap p^{-1}(N \cdot \theta)$.

In particular, in the nonempty intersection case, $\Omega_{\pi} \cap p^{-1}(N \cdot \theta)$ is connected and

$$
\operatorname{dim} \Omega_{\pi} \cap p^{-1}(N \cdot \theta)=\operatorname{dim} G_{\theta} N / G_{\varphi} .
$$

Now suppose we are in the multiplicity-free case of formula (3). Then $G_{\gamma} / N$ is abelian and the Mackey obstruction vanishes. Let $\varphi \in p^{-1}(\theta)$. Set $\xi=\left.\varphi\right|_{g_{\theta}}$. Since $G_{\gamma} / N \simeq G_{\theta} / N_{\theta}$ is abelian and there is no obstruction to extending the character $\chi_{\theta}$ from $N_{\theta}$ to $G_{\theta}$, it must be that $\left(\mathfrak{g}_{\theta}\right)_{\xi}=\mathfrak{g}_{\theta}$ for any $\xi \in \mathfrak{g}_{\theta}^{*}$, $\left.\xi\right|_{\mathfrak{n}_{\theta}}=\left.\theta\right|_{\mathfrak{n}_{\theta}}$. But it is also true that $\left(\mathfrak{g}_{\theta}\right)_{\xi}=\mathfrak{g}_{\varphi}+\mathfrak{n}_{\theta}($ see $[11$, p. 271, (v)] or [13, Corollary 3.5]). Therefore $G_{\theta}=G_{\varphi} N_{\theta}$. But then $G \cdot \varphi \cap p^{-1}(N \cdot \theta)=$ $G_{\theta} N \cdot \varphi=N G_{\theta} \cdot \varphi=N \cdot \varphi$, and so the direct integral in formula (2) is also multiplicity-free.

On the other hand, suppose we are in the uniformly infinite case of formula (3). Then either $G_{\gamma} / N$ is nonabelian, or it is abelian and the multiplier $\omega_{\gamma}$ is nontrivial. In either case, the generic (projective) representations of $G_{\gamma} / N$ are infinite-dimensional. Therefore, generically, the functionals $\xi \in \mathfrak{g}_{\theta}^{*},\left.\xi\right|_{\mathfrak{n}_{\theta}}=$ $\left.\theta\right|_{\mathfrak{n}_{\theta}}$, have the property that $\left(\mathfrak{g}_{\theta}\right)_{\xi} \varsubsetneqq \mathfrak{g}_{\theta}$. This time then $\operatorname{dim}\left(\mathfrak{g}_{\varphi}+\mathfrak{n}_{\theta}\right)<\operatorname{dim} \mathfrak{g}_{\theta}$ for generic $\varphi \in p^{-1}(\theta)$. Hence

$$
\begin{aligned}
\operatorname{dim} G \cdot \varphi \cap p^{-1}(N \cdot \theta) & =\operatorname{dim} G_{\theta} N / G_{\varphi}>\operatorname{dim} G_{\varphi} N / G_{\varphi} \\
& =\operatorname{dim} N / N_{\varphi}=\operatorname{dim} N \cdot \varphi .
\end{aligned}
$$

That is, the multiplicity in formula (2) is generically equal to $+\infty$. This concludes the proof of equal multiplicity. (Note we omit the proof that the multiplicity-free case is equivalent to the second equality in the statement of the theorem. This is absolutely identical to Proposition 3.4.)

It remains only to show the measure classes in formulas (2) and (3) are the same. In fact I will write down an underlying isomorphism of the measure spaces that carries one measure onto the other. First of all, by the final paragraph in $\S 3$, we know that $\mu_{G, N}^{\gamma}$ is the push-forward of Lebesgue measure on $p^{-1}(\theta)$. On the other hand, if we set $\tilde{\mathfrak{g}}_{\theta}^{*}=\left\{\xi \in \mathfrak{g}_{\theta}^{*}:\left.\xi\right|_{\mathfrak{n}_{\theta}}=\left.\theta\right|_{\mathfrak{n}_{\theta}}\right\}$, then Plancherel measure on $\left(G_{\gamma}^{\widehat{\gamma}} / N\right)^{\bar{\omega}_{\bar{\gamma}}}$ is nothing more than the push-forward of Lebesgue mesaure on $\tilde{\mathfrak{g}}_{\theta}^{*}$ under the map $\tilde{\mathfrak{g}}_{\theta}^{*} \rightarrow \tilde{\mathfrak{g}}_{\theta}^{*} / G_{\theta}$. We use [12, p. 360]. Consider the map $\xi \rightarrow G \cdot \varphi_{\xi}$, $\tilde{\mathfrak{g}}_{\theta}^{*} \rightarrow \mathfrak{g}^{*} / G$, where $\varphi_{\xi}=$ any extension to $\mathfrak{g}$ of the linear functional on $\mathfrak{g}_{\theta}+\mathfrak{n}$ which agrees on $\mathfrak{n}$ with $\theta$ and on $\mathfrak{g}_{\theta}$ with $\xi$. If $\varphi_{\xi}^{\prime}$ is another extension, then $\left.\varphi_{\xi}^{\prime}\right|_{g_{\theta+\mathrm{n}}}=\left.\varphi_{\xi}\right|_{g_{1 / n}+\mathrm{n}} \Rightarrow \varphi_{\xi}^{\prime}=n_{\theta} \cdot \varphi_{\xi}, n_{\theta} \in N_{\theta}$. So $\xi \rightarrow \varphi_{\xi}$ is well defined. The map 
is obviously surjective on $G \cdot p^{-1}(\theta) / G$. Finally, we have

$$
G \cdot \varphi_{\xi}=G \cdot \varphi_{\xi_{1}} \Leftrightarrow G_{\theta} \cdot \xi=G_{\theta} \cdot \xi_{1} .
$$

Indeed if $g_{\theta} \cdot \xi=\zeta_{1}$, then $\left.g_{\theta} \cdot \varphi_{\xi}\right|_{g_{\theta}+\mathfrak{n}}=\left.\varphi_{\xi_{1}}\right|_{g_{\theta}+\mathfrak{n}} \Rightarrow \varphi_{\xi_{1}}=n_{\theta} g_{\theta} \cdot \varphi_{\xi}$. Conversely if $g \cdot \varphi_{\xi}=\varphi_{\xi_{1}}$, then $\theta=\left.\varphi_{\xi_{1}}\right|_{\mathfrak{n}}=\left.g \cdot \varphi_{\xi}\right|_{\mathfrak{n}}=g \cdot \theta$, i.e., $g \in G_{\theta}$. The equality of the measure classes-both push-forwards of Lebesgue measure-is now evident.

Next we turn our attention to restrictions. The analog of Theorem 6.1 is

Theorem 6.2. Let $\varphi \in \mathfrak{g}^{*}, \pi=\pi_{\varphi} \in \hat{G}$ the corresponding representation, $p=$ $p_{\mathfrak{g}, \mathfrak{n}}$. Then

$$
\left.\pi_{\varphi}\right|_{N}=\int_{p(G \cdot \varphi) / N}^{\oplus} n_{\varphi, \theta} \gamma_{\theta} d \lambda_{N, G}^{\pi}(\theta),
$$

where $\lambda_{N, G}^{\pi}$ is the push-forward of the canonical measure on $G \cdot \varphi$ under $G \cdot \varphi \rightarrow$ $p(G \cdot \varphi) \rightarrow p(G \cdot \varphi) / N$. Moreover, the multiplicity function

$$
n_{\varphi, \theta}=\# N \text {-orbits in } p^{-1}(N \cdot \theta) \cap G \cdot \varphi
$$

is identically 1 or $+\infty$ according to the same conditions as Theorem 6.1 (except generic on $\theta$ instead of $\varphi$ ).

Proof. We start with the Mackey machine as in Theorem 6.1. Set $\theta=\left.\varphi\right|_{\mathfrak{n}}$, $\gamma=\gamma_{\theta} \in \hat{N}$, and $\tilde{\gamma}$ an extension determining the Mackey cocycle $\omega_{\gamma}$. There is a unique $\sigma \in\left(G_{\gamma} / N\right)^{\bar{\omega}_{\text {, }}}$, which is determined by $\xi=\left.\varphi\right|_{\mathfrak{g}_{\theta}}$ (see [12]), so that

$$
\pi_{\varphi}=\pi_{\gamma, \sigma}=\operatorname{Ind}_{G,}^{G} \sigma \otimes \tilde{\gamma} .
$$

Moreover, by the work in [10] we know

$$
\left.\pi_{\gamma, \sigma}\right|_{N}=\int_{G / G_{\gamma^{\prime}}}^{\oplus}(\operatorname{dim} \sigma) g \cdot \gamma d \dot{g} .
$$

Now, exactly as before, $G_{\gamma} / N$ is exponential solvable and the generic representations of $\left(G_{\gamma} / N\right)^{\bar{\omega}_{\gamma}}$ are all of the same dimension, i.e., 1 or $\infty$. The former occurs iff $G_{\gamma} / N$ is abelian and $\omega_{\gamma}$ is trivial. We must show the identity of the direct integral decompositions (4) and (5).

The spectra are clearly the same. Indeed the spectrum in (4) is $p(G \cdot \varphi) / N=$ $G \cdot \theta / N \subset \mathfrak{n}^{*} / N$, which agrees with $G \cdot \gamma \subset \hat{N}$ under the Kirillov identification of $\mathrm{n}^{*} / N$ with $\hat{N}$. Next come the multiplicities. But for this it is virtually sufficient to cite the proof of Theorem 6.1. We saw there that

$$
\begin{aligned}
& \# N \text {-orbits on } p^{-1}(N \cdot \theta) \cap G \cdot \varphi=1 \\
& \quad \Leftrightarrow G_{\theta} N=G_{\varphi} N \Leftrightarrow G_{\theta}=G_{\varphi} N_{\theta} \\
& \quad \Leftrightarrow G_{\gamma} / N \text { is abelian and } \omega_{\gamma} \text { is trivial; }
\end{aligned}
$$


and in the contrary case

$$
\begin{aligned}
& \# N \text {-orbits on } p^{-1}(N \cdot \theta) \cap G \cdot \varphi=+\infty \\
& \Leftrightarrow \operatorname{dim} N \cdot \varphi<\operatorname{dim} G \cdot \varphi \cap p^{-1}(N \cdot \theta) \\
& \Leftrightarrow \text { the generic representations } \sigma \text { of }\left(G_{\gamma} / N\right)^{\bar{\omega}_{i /}} \text { are } \\
& \quad \infty \text {-dimensional. }
\end{aligned}
$$

Finally, the spectral measures are exactly the same-indeed

$$
p(G \cdot \varphi) / N=G \cdot \theta / N=G / G_{\theta} N=G / G_{\gamma}
$$

and the canonical quotient measure classes are identical.

\section{CONORMAL COSET SPACES}

We are still interested in decomposing induced representations $\operatorname{Ind}_{H}^{G} \nu$ but, unlike the situation in the last section, in which $H$ was normal, we assume now $H$ is conormal. This means that $G$ is a semidirect product $G=H N$ where $N$ is normal. The main impetus for the study of conormally induced representations has come from two sources. The first is the study of symmetric spaces as, for example, in [16 or 20]. The second-and more to the point here-is the study of exponential solvable homogeneous spaces. As mentioned in the introduction, much of the argumentation of $\S \S 3,4$ really depends on the nilpotency of $G$ only to ensure the existence of the codimension 1 normal subgroup $N$. If $N$ exists a priori, the technique of Part I can be employed. When does $N$ fail to exist? The typical situation is exemplified by the $a x+b$ group

$$
G=\left\{\left(\begin{array}{ll}
a & b \\
0 & 1
\end{array}\right): a>0, b \in \mathbf{R}\right\} \quad \text { with } H=\left\{\left(\begin{array}{ll}
a & 0 \\
0 & 1
\end{array}\right): a>0\right\} .
$$

$H$ is not contained in any proper normal subgroup of $G$. But this is a typical conormal subgroup, and the understanding of $\operatorname{Ind}_{H}^{G} \nu$ is critical to the study of exponential solvable homogeneous spaces.

Now the same thread that is present in Kirillov [9], Auslander-Kostant [1], Pukanszky [19], Duflo [6, 7], and Lipsman [12, 13] is present here. Namely, we seek an orbital description of the harmonic analysis of $L^{2}(G / H)$. The main ingredients of that analysis are given by the Mackey machine. For these Mackey parameters to disappear from the final picture, we must understand the interplay between the Mackey and orbital parameters (as we did, e.g., in §6). In this section we give the main Mackey machine result for conormally induced representations. Its interplay with orbits will be considered in future publications. One simplification we will make now-the irreducible representations of exponential solvable groups are monomial, so we will assume the inducing representation is a character.

Let $G=H N$ be a semidirect product of locally compact groups, $N$ normal and type I. The representations of $G$ produced by the Mackey machine are as 
follows. Let $\gamma \in \hat{N}, H_{\gamma}$ the stability group. Let $\tilde{\gamma}$ be any extension of $\gamma$ to $H_{\gamma}$ satisfying

$$
\tilde{\gamma}(h) \gamma\left(h^{-1} n h\right)=\gamma(n) \tilde{\gamma}(h), \quad n \in N, h \in H_{\gamma} .
$$

$\tilde{\gamma}$ is not uniquely defined, but the Mackey cocycle (class) defined by

$$
\tilde{\gamma}\left(h_{1}\right) \tilde{\gamma}\left(h_{2}\right)=\omega_{\gamma}\left(h_{1}, h_{2}\right) \tilde{\gamma}\left(h_{1} h_{2}\right), \quad h_{i} \in H_{\gamma},
$$

is uniquely determined. We may assume $\omega_{\gamma}$ is a normalized multiplier-i.e., $\omega_{\gamma}\left(h, h^{-1}\right)=1$. The Mackey machine representations are given by

$$
\pi_{\gamma, \sigma}=\operatorname{Ind}_{H_{\gamma, N}}^{G} \sigma \otimes \tilde{\gamma} \times \gamma, \quad \sigma \in \hat{H}_{\gamma}^{\bar{\omega}_{\gamma}} .
$$

We have

$$
\pi_{\gamma, \sigma} \simeq \pi_{\gamma^{\prime} \sigma^{\prime}} \Leftrightarrow \exists g \in G, g \cdot \gamma \simeq \gamma^{\prime}, g \cdot \sigma \simeq \sigma^{\prime} .
$$

In case $\hat{N} / H$ is countably separated and all the little group duals $\hat{H}_{\gamma}^{\bar{\omega}_{\text {, }}}$ are type I, we obtain all the irreducible representations of $G$ this way.

Theorem 7.1. Suppose $N$ is unimodular, $\hat{N} / H$ is countably separated, and $\tilde{\gamma}$ is type I for $\mu_{N}$-a.a $\gamma \in \hat{N}$. Let

$$
\tilde{\gamma}=\int_{\hat{H}_{\gamma}^{\omega} \gamma}^{\oplus} n_{\gamma}(\sigma) \sigma d \mu_{\gamma}(\sigma)
$$

be the unique direct integral decomposition of $\tilde{\gamma}$. Then

$$
\operatorname{Ind}_{H}^{G} 1=\int_{\hat{N} / H}^{\oplus} \int_{\hat{H}_{\gamma}^{\bar{\omega} \gamma}}^{\oplus} n_{\gamma}(\bar{\sigma}) \pi_{\gamma, \sigma} d \mu_{\gamma}(\sigma) d \dot{\mu}_{N}(\gamma),
$$

where $\dot{\mu}_{N}$ is the push-forward of the Plancherel measure $\mu_{N}$ on $\hat{N}$.

Proof. First we observe that the final result does not depend on the choice of the Mackey extensions $\tilde{\gamma}$. Indeed any other extension $\tilde{\gamma}_{1}$ that satisfies equation (6) is of the form $\tilde{\gamma}_{1}=\chi \tilde{\gamma}, \chi$ a unitary character of $H_{\gamma}$. Note that the notation $\pi_{\gamma, \sigma}$ is slightly abusive $-\pi_{\gamma, \tilde{\gamma}, \sigma}$ is more accurate. It is clear that

Furthermore if

$$
\pi_{\gamma, \tilde{\gamma}, \sigma} \simeq \pi_{\gamma, \tilde{\gamma}_{1}, \bar{\chi} \sigma} \quad \text { if } \tilde{\gamma}_{1}=\chi \tilde{\gamma} .
$$

then

$$
\tilde{\gamma}=\int_{\hat{H}_{;} \omega_{r}}^{\oplus} n_{\tilde{\gamma}}(\sigma) \sigma d \mu_{\gamma}(\sigma)
$$

$$
\chi \tilde{\gamma}=\int_{\hat{H}_{\gamma}^{\omega} \gamma}^{\oplus} n_{\tilde{\gamma}}(\sigma) \chi \sigma d \mu_{\gamma}(\sigma)=\int_{\hat{H}_{\gamma}^{\omega} \gamma} n_{\tilde{\gamma}}(\bar{\chi} \sigma) \sigma d \mu_{\chi \gamma}(\sigma) .
$$

That is,

$$
n_{\chi \tilde{\gamma}}(\sigma)=n_{\tilde{\gamma}}(\bar{\chi} \sigma), \quad \sigma \in \hat{H}_{\gamma}^{\bar{\omega}} \gamma .
$$

It follows that the expression

$$
\int_{\hat{H}_{\gamma}^{\bar{\omega}},}^{\oplus} n_{\tilde{\gamma}}(\bar{\sigma}) \pi_{\gamma, \tilde{\gamma}, \sigma} d \mu_{\gamma}(\sigma)
$$


is independent of the choice of $\tilde{\gamma}$. Indeed

$$
n_{\hat{\gamma}_{1}}(\bar{\sigma}) \pi_{\gamma, \hat{\gamma}_{1}, \sigma}=n_{\chi \hat{\gamma}}(\bar{\sigma}) \pi_{\gamma, \chi \tilde{\gamma}, \sigma}=n_{\hat{\gamma}}(\overline{\chi \sigma}) \pi_{\gamma, \tilde{\gamma}, \chi \sigma},
$$

so that the change of variable $\chi \sigma=\rho$ effects the proof.

Next we square away a few modular functions. To conform to [10], we put all group actions on the right-e.g., $n \cdot h=h^{-1} n h,(\gamma \cdot h)(n)=\gamma\left(n \cdot h^{-1}\right)$, etc. We use $\Delta$ for modular functions, e.g.,

$$
\Delta_{H}(x) \int_{H} f(x h) d h=\int_{H} f(h) d h, \quad f \in C_{c}(H),
$$

where $d h$ =right Haar measure on $H$. We also define the modulus $q$ for the action of $H$ on $N$ by

$$
q(h) \int_{N} f\left(h n h^{-1}\right) d n=\int_{N} f(n) d n, \quad f \in C_{c}(N) .
$$

It is an easy exercise to verify that

$$
\Delta_{G}(n h)=\Delta_{G}(h)=q(h) \Delta_{H}(h) .
$$

Now since $N$ is unmodular and type $\mathrm{I}$, it has a nice Plancherel theorem: there are a measurable field of Hilbert spaces $\gamma \rightarrow \mathscr{H}_{\gamma}$, a measurable field of irreducible unitary representations $\gamma \rightarrow \Gamma_{\gamma} \in \gamma$ on $\mathscr{H}_{\gamma}$, and a positive Borel measure $\mu_{N}$ so that

$$
f \rightarrow \Gamma_{\gamma}(f)=\int_{N} f(n) \Gamma_{\gamma}(n) d n, \quad f \in C_{c}(N),
$$

extends to a unitary operator

$$
\mathscr{F}: L^{2}(N) \rightarrow \int_{\hat{N}}^{\oplus} \mathscr{H} \mathscr{S}\left(\mathscr{H}_{\gamma}\right) d \mu_{N}(\gamma)
$$

intertwining $\lambda \otimes \rho$ with $\int^{\oplus} \Gamma_{\gamma} \otimes \bar{\Gamma}_{\gamma} d \mu_{N}(\gamma)$. Here $\lambda$ and $\rho$ denote the left and right regular representations, and $\mathscr{H} \mathscr{S}\left(\mathscr{H}_{\gamma}\right) \simeq \mathscr{H}_{\gamma} \otimes \overline{\mathscr{H}}_{\gamma}$ is the Hilbert space of Hilbert-Schmidt operators on $\mathscr{H}_{\gamma}$. As usual the identification of $\mathscr{H} \mathscr{S}\left(\mathscr{H}_{\gamma}\right)$ with $\mathscr{H}_{\gamma} \otimes \overline{\mathscr{H}}_{\gamma}$ is via $T_{\xi \otimes \eta}(\zeta)=(\zeta, \eta) \xi, \xi, \eta, \zeta \in \mathscr{H}_{\gamma}$, extended by linearity and continuity. It is routine to check that for any bounded operator $D$ on $\mathscr{H}_{\gamma}$ we have

$$
D T_{\xi \otimes \eta}=T_{D \xi \otimes \eta}, \quad T_{\xi \otimes \eta} D=T_{\xi \otimes D^{*} \eta} .
$$

Equation (7) says that Haar measure on $N$ is relatively invariant under $H$ with modulus $q$. After transforming by $\mathscr{F}$, it must be that Plancherel measure $\mu_{N}$ is also relatively invariant, this time with modulus $q^{-1}$-i.e.,

$$
q(h)^{-1} \int_{\hat{N}}\left\|\hat{f}\left(\gamma \cdot h^{-1}\right)\right\|_{2}^{2} d \mu_{N}(\gamma)=\int_{\hat{N}}\|\hat{f}(\gamma)\|_{2}^{2} d \mu_{N}(\gamma) .
$$

Next we disintegrate $\left(\hat{N}, \mu_{N}\right)$ under the action of $H$ (see [10]). Fix a pseudoimage $\dot{\mu}_{N}$ (i.e., a push-forward) of $\mu_{N}$ under $\hat{N} \rightarrow \hat{N} / H$. It is standard 
Borel measure since $\hat{N} / H$ is countably separated. Then by [10, p. 128] there exist uniquely determined relatively invariant measures $d \bar{h}$ on $H / H_{\gamma}$ such that

$$
\int_{\hat{N}} F(\gamma) d \mu_{N}(\gamma)=\int_{\hat{N} / H} \int_{H / H_{\gamma}} F(\gamma \cdot h) d \bar{h} d \dot{\mu}_{N}(\bar{\gamma})
$$

(the shorthand is $\bar{\gamma}=\gamma \cdot H$ ). The modulus of (a.a. of) the relatively invariant measures $d \bar{h}$ must be the same as the modulus of $\mu_{N}\left[10\right.$, p. 108], i.e., $q^{-1}$. But it is well known that the homogeneous space $H / H_{\gamma}$ has a relatively invariant measure (with modulus $q_{\gamma}$ ) iff the function $h_{\gamma} \rightarrow \Delta_{H}\left(h_{\gamma}\right) \Delta_{H_{\gamma}}\left(h_{\gamma}\right)^{-1}$ satisfies

$$
q_{\gamma}\left(h_{\gamma}\right)=\Delta_{H}\left(h_{\gamma}\right) \Delta_{H_{\gamma}}\left(h_{\gamma}\right)^{-1}, \quad h_{\gamma} \in H_{\gamma} .
$$

In particular, the latter extends to a continuous character of $H$ to $\mathbf{R}_{+}^{\times}$which agrees with $q^{-1}$.

Now with all these preliminaries set, we can get to the details of the actual proof. We begin by realizing the induced representation $R=\operatorname{Ind}_{H}^{G} 1$ on $L^{2}(N)$. We can do this since $G=H N$. Indeed, using the standard formulas for an induced representation [10] and restricting the function space to $N$, we obtain

$$
\begin{aligned}
& R(n) f\left(n_{1}\right)=f\left(n_{1} n\right), \\
& R(h) f\left(n_{1}\right)=q(h)^{-1 / 2} f\left(h^{-1} n_{1} h\right), \quad f \in L^{2}(N) .
\end{aligned}
$$

Next we transfer the action of $R$ to $\int_{\hat{N}}^{\oplus} \mathscr{H} \mathscr{S}(\mathscr{H}) d \mu_{N}(\gamma)$ via $\mathscr{F}$. But before doing that we must make a selection. For each pair $h \in H, \gamma \in \hat{N}$ we select a unitary operator

so that

$$
\tilde{\gamma}(h): \mathscr{H}_{\gamma \cdot h} \rightarrow \mathscr{H}_{\gamma}
$$

$$
\Gamma_{\gamma}\left(h n h^{-1}\right)=\tilde{\gamma}(h) \Gamma_{\gamma \cdot h}(n) \tilde{\gamma}(h)^{-1}, \quad n \in N, h \in H .
$$

This can be done in a measurable way. Note that when $h \in H_{\gamma}$, then $\gamma \cdot h=\gamma$ and the operator $\tilde{\gamma}(h)$ may be taken to be a Mackey extension. Also we leave it to the reader to check the existence of a measurable function $\omega_{\gamma}$ on $H \times H$ so that

$$
\tilde{\gamma}(h k)=\tilde{\gamma}(h)(\gamma \cdot h)^{\sim}(k) \omega_{\gamma}(h, k), \quad h, k \in H .
$$

Thus $\left.\omega_{\gamma}\right|_{H_{i} \times H_{i}}$ is the Mackey multiplier.

Now we write $\left\{T_{\gamma}\right\}$ to denote an element of $\int^{\oplus} \mathscr{H} \mathscr{S}\left(\mathscr{H}_{\gamma}\right)$. These are Hilbert Schmidt operator fields over $\hat{N}$, specified $\mu_{N}$-a.e. A simple computation reveals that if $\hat{R}=\mathscr{F} \circ R \circ \mathscr{F}^{-1}$, then

$$
\hat{R}(n)\left\{T_{\gamma}\right\}=\left\{T_{\gamma} \Gamma_{\gamma}(n)^{-1}\right\}, \quad \hat{R}(h)\left\{T_{\gamma}\right\}=\left\{q(h)^{1 / 2} \tilde{\gamma}(h) T_{\gamma \cdot h} \tilde{\gamma}(h)^{-1}\right\} .
$$

But from the disintegration of $\left(\hat{N}, \mu_{N}\right)$ under the action of $H$ we have

$$
\int_{\hat{N}}^{\oplus} \mathscr{H} \mathscr{S}\left(\mathscr{H}_{\gamma}\right) d \mu_{N}(\gamma)=\int_{\hat{N} / H}^{\oplus} \int_{H / H_{i}}^{\oplus} \mathscr{H} \mathscr{S}\left(\mathscr{H}_{\gamma \cdot h}\right) d \bar{h} d \dot{\mu}_{N}(\bar{\gamma}) \text {. }
$$


It is completely evident from the formulas (10) that the inside integrals are $G$ invariant (under $\hat{R}$ ). Thus to prove the theorem, it is enough to prove that the action of $G$ via $\hat{R}$ on

$$
\int_{\hat{H} / H_{i}}^{\oplus} \mathscr{H} \mathscr{S}\left(\mathscr{H}_{\gamma \cdot h}\right) d \bar{h}
$$

is unitarily equivalent to the representation

$$
\int_{\hat{H}_{;}^{\bar{\omega}_{i}}}^{\oplus} n_{\gamma}(\bar{\sigma}) \pi_{\gamma, \sigma} d \mu_{\gamma}(\sigma) .
$$

In fact we shall write down an explicit intertwining operator. But because of the way we set up the structure, it will be more convenient to conjugate equation (11). So to exhibit the operator, we return to the Mackey machine and reparametrize slightly. Write

$$
\pi_{\bar{\gamma}, \sigma}=\operatorname{Ind}_{H_{\gamma}, N}^{G} \sigma \otimes \tilde{\bar{\gamma}} \times \bar{\gamma}, \quad \bar{\gamma} \text { the conjugate representation, } \sigma \in \hat{H}_{\gamma}^{\omega_{\gamma}} .
$$

We may realize this induced representation in the space of Borel functions

$$
f: H \rightarrow \mathscr{H}_{\sigma} \otimes \overline{\mathscr{H}}_{\gamma}
$$

which satisfy

$$
\begin{aligned}
& f\left(h_{1} h\right)=\left[\sigma\left(h_{1}\right) \otimes \tilde{\bar{\gamma}}\left(h_{1}\right)\right] f(h), \quad h \in H, h_{1} \in H_{\gamma}, \\
& \int_{H / H_{i}}\|f\|^{2} d \bar{h}<\infty
\end{aligned}
$$

via the action

$$
\begin{gathered}
\pi_{\bar{\gamma}, \sigma}(k) f(h)=f(h k) q_{\gamma}(k)^{-1 / 2}, \quad h, k \in H, \\
\pi_{\bar{\gamma}, \sigma}(n) f(h)=\Gamma_{\gamma}\left(h n h^{-1}\right) f(h), \quad h \in H, n \in N \quad \text { (see [10]). }
\end{gathered}
$$

We call the above space $\mathscr{H}_{\bar{\gamma}, \sigma}$. Now we define an intertwining operator $\Phi$ that maps

$$
\Phi: \int_{H / H_{i}}^{\oplus} \mathscr{H} \mathscr{S}\left(\mathscr{H}_{\gamma \cdot h}\right) d \bar{h} \rightarrow \int_{\hat{H}^{\left(\omega_{j},\right.}}^{\oplus} n_{\bar{\gamma}}(\sigma) \mathscr{H}_{\bar{\gamma}, \sigma} d \mu_{\gamma}(\sigma) .
$$

In order to define $\Phi$ we invoke the hypothesis of the theorem to know there is a unitary operator

$$
\Phi_{\gamma}: \mathscr{H}_{\gamma} \rightarrow \int_{\hat{H}^{\omega_{\gamma}}}^{\oplus} \mathscr{H}_{\gamma}(\sigma) d \mu_{\gamma}(\sigma)
$$

which intertwines $\tilde{\gamma}$ and $\int_{\hat{H}^{\omega_{j} ;}}^{\oplus} n_{\gamma}(\sigma) \sigma d \mu_{\gamma}(\sigma)$. That is, for $\xi \in \mathscr{H}_{\gamma}, \Phi_{\gamma}(\xi)$ is a measurable field of vectors $\left\{\Phi_{\gamma}^{\sigma}(\xi)\right\}_{\sigma \in \hat{H}^{\left(\omega_{i}\right.}}$, where $\Phi_{\gamma}^{\sigma}(\xi) \in \mathscr{H}_{\gamma}(\sigma)$-a space on which $H_{\gamma}$ acts by $n_{\gamma}(\sigma) \sigma\left(\mu_{\gamma}\right.$-a.e. $)$. Thus

$$
\Phi_{\gamma}^{\sigma}(\tilde{\gamma}(h) \xi)=n_{\gamma}(\sigma) \sigma(h) \Phi_{\gamma}^{\sigma}(\xi), \quad h \in H_{\gamma} .
$$

Next, to define $\Phi$ we set

$$
\Phi:\left\{T_{\gamma \cdot h}\right\} \rightarrow\left\{f_{\sigma}^{T}\right\}
$$


where

$$
f_{\sigma}^{T}(h)=\Phi_{\gamma}^{\sigma} \tilde{\gamma}(h) T_{\gamma \cdot h} \tilde{\gamma}(h)^{-1}
$$

$h \rightarrow f_{\sigma}^{T}(h)$ is a Borel function with values in Hilbert-Schmidt operators from $\mathscr{H}_{\gamma}$ to $\mathscr{H}_{\gamma}(\sigma)$, i.e., an element of $\mathscr{H}_{\gamma}(\sigma) \otimes \overline{\mathscr{H}}_{\gamma}$. (We use equation (8) to identify $\mathscr{A} \otimes \overline{\mathscr{B}} \simeq \mathscr{H} \mathscr{S}(\mathscr{B}, \mathscr{A})$ for Hilbert spaces $\mathscr{A}, \mathscr{B}$.) Moreover

$$
\begin{aligned}
f_{\sigma}^{T}\left(h_{\gamma} h\right) & =\Phi_{\gamma}^{\sigma} \tilde{\gamma}\left(h_{\gamma} h\right) T_{\gamma \cdot h_{\gamma} h} \tilde{\gamma}\left(h_{\gamma} h\right)^{-1} \\
& =\Phi_{\gamma}^{\sigma} \tilde{\gamma}\left(h_{\gamma}\right) \tilde{\gamma}(h) T_{\gamma \cdot h} \tilde{\gamma}(h)^{-1} \tilde{\gamma}\left(h_{\gamma}\right)^{-1} \\
& =\left[n_{\gamma}(\sigma) \sigma \otimes \tilde{\gamma}\right]\left(h_{\gamma}\right) f_{\sigma}^{T}(h),
\end{aligned}
$$

because of the intertwining property of $\Phi_{\gamma}^{\sigma}$ and the identification in the second of equations (8). It is clear, virtually by definition, that $\left\|f_{\sigma}^{T}(\cdot)\right\|_{2}$ is squareintegrable on $H / H_{\gamma}$. Thus the operator $\Phi$ is well defined. We must show that it is unitary and that it intertwines the associated group actions. Unitarity is clear-by definition and the fact that the $\tilde{\gamma}(h)$ are unitary operators. We show the intertwining property by separating out the $N$ and $H$ actions. We compute

$$
\begin{aligned}
f_{\sigma}^{n \cdot T}(h) & =\Phi_{\gamma}^{\sigma} \tilde{\gamma}(h)(\hat{R}(n) T)_{\gamma \cdot h} \tilde{\gamma}(h)^{-1} \\
& =\Phi_{\gamma}^{\sigma} \tilde{\gamma}(h) T_{\gamma \cdot h} \Gamma_{\gamma \cdot h}(n)^{-1} \tilde{\gamma}(h)^{-1} \\
& =f_{\sigma}^{T}(h) \tilde{\gamma}(h) \Gamma_{\gamma \cdot h}(n)^{-1} \tilde{\gamma}(h)^{-1} \\
& =f_{\sigma}^{T}(h) \Gamma_{\gamma}\left(h n h^{-1}\right)=n \cdot f_{\sigma}^{T}
\end{aligned}
$$

by equation (12b) and the second of equations (8) again. Also

$$
\begin{aligned}
f_{\sigma}^{k \cdot T}(h) & =\Phi_{\gamma}^{\sigma} \tilde{\gamma}(h)(\hat{R}(k) T)_{\gamma \cdot h} \tilde{\gamma}(h)^{-1} \\
& =\Phi_{\gamma}^{\sigma} \tilde{\gamma}(h) q(k)^{1 / 2}(\gamma \cdot h)^{\sim}(k) T_{\gamma \cdot h k}(\gamma \cdot h)^{\sim}(k)^{-1} \tilde{\gamma}(h)^{-1} \\
& =q(k)^{1 / 2} \Phi_{\gamma}^{\sigma} \tilde{\gamma}(h k) T_{\gamma \cdot h k} \tilde{\gamma}(h k)^{-1} \\
& =q(k)^{1 / 2} f_{\sigma}^{T}(h k)=k \cdot f_{\sigma}^{T}
\end{aligned}
$$

by equation (12a) and the equality of $q_{\gamma}$ with $q^{-1}$. Note we also used (9) (the $\omega_{\gamma}$ 's cancel out in the application). Q.E.D.

Note. It is a cute exercise to apply Theorem 7.1 to the situation where $G=S \times S$, $H=\Delta^{G}=\{(s, s): s \in S\}, N=\{(1, s): s \in S\}, S$ a unimodular type I group.

Theorem 7.1 may be extended by allowing the inducing representation to be any character, not just the identity. The proof is basically the same and so I shall not repeat it. Here is the precise result.

Theorem 7.2. Suppose $N$ is unimodular, $\hat{N} / H$ is countably separated, and $\tilde{\gamma}$ is type I (a.e.). Let

$$
\tilde{\gamma}=\int_{\hat{H}_{i}^{\omega}}^{\oplus} n_{\gamma}(\sigma) \sigma d \mu_{\gamma}(\sigma)
$$


as in Theorem 7.1. Let $\chi \in \hat{H}$ be a character and set $\chi_{\gamma}=\chi \mid H_{\gamma}$. Then

$$
\operatorname{Ind}_{H}^{G} \chi=\int_{\hat{N} / H}^{\oplus} \int_{\hat{H}_{;}^{\bar{\omega}_{\gamma}}}^{\oplus} n_{\gamma}\left(\chi_{\gamma} \bar{\sigma}\right) \pi_{\gamma, \sigma} d u_{\gamma}(\sigma) d \dot{\mu}_{N}(\gamma),
$$

the notation interpreted exactly as in Theorem 7.1 .

Remark 7.3. Viewed from the Mackey perspective, these theorems say that to describe the decomposition of the induced representation, it is enough to understand the decomposition of $\tilde{\gamma}$. That in itself is a very interesting problem. The most interesting case is when $N$ is simply connected nilpotent. If $H$ is a group of automorphisms of $N$ preserving some $\gamma \in \hat{N}$, the problem is to describe the representation $\tilde{\gamma}$ of $H$. The question has been studied extensively for $H$ semisimple (the Weil or oscillator representation). It is also interesting to ponder other categories of $H$ (e.g., $H$ nilpotent or exponential solvable) and what the "orbital description" of $\tilde{\gamma}$ might look like. In our next publication (on exponential solvable homogeneous spaces) we shall have to deal with $H$ abelian. Other questions one may ask in general are: when is $\tilde{\gamma}$ of finite multiplicity? multiplicity free? quasi-equivalent to the regular representation?

\section{EXAMPLES}

In this last section we present various examples of direct integral decompositions of nonnilpotent homogeneous spaces. For simplicity we keep to $\operatorname{Ind}_{H}^{G} 1$, i.e., the quasi-regular representation. Our primary concern here is to indicate how it may be possible to generalize Theorem 2.2 to nonnilpotent groups $G$. In fact, we expect it to go over unchanged to exponential solvable groups and in "some modified form" to more general groups. The examples presented here may be thought of as clues or signposts to the as yet unspecified final formulation.

I shall present only the direct integral formulas here-not their detailed derivations. This is to keep down the length of the exposition.

(i) The split oscillator. Let $G=\exp \mathfrak{g}$, where $\mathfrak{g}$ is the 4-dimensional exponential solvable Lie algebra having generators $W, X, Y, Z$ satisfying bracket relations

$$
[W, X]=X, \quad[W, Y]=-Y, \quad[X, Y]=Z .
$$

A cross section for the generic orbits in $\mathfrak{g}^{*}$ is given by

$$
\mathscr{C}=\left\{\varphi=\omega W^{*}+\zeta Z^{*}: \zeta \neq 0, \omega \in \mathbf{R}\right\} .
$$

Let $H=\exp \mathbf{R} W$. Then every generic orbit meets $\mathfrak{h}^{\perp}$, and if we denote a push-forward of Lebesgue measure by a dot, we have

$$
\operatorname{Ind}_{H}^{G} 1=\int_{\mathfrak{h}^{\perp} / H}^{\oplus} \pi_{\varphi} d \dot{\varphi} \text {. }
$$

Furthermore

$$
n_{\varphi}=\# H \text {-orbits on } G \cdot \varphi \cap \mathfrak{h}^{\perp}=2, \quad \text { for all } \varphi_{(\omega, \zeta)} \in \mathscr{C}, \omega \neq 0 .
$$


$G$ and $H$ are algebraic exponential solvable, and in fact we shall prove all of Theorems 1.5, 2.2, 3.1, 4.1 for such groups in our next paper in this series. (Note $H$ is conormal.)

(ii) Grélaud's example. Let $G=\exp \mathfrak{g}$, where $\mathfrak{g}$ is the 3-dimensional exponential solvable Lie algebra having generators $W, X, Y$ satisfying bracket relations

$$
[W, X]=X-Y, \quad[W, Y]=X+Y .
$$

The generic coadjoint orbits are 2-dimensional. Let $H=\exp \mathbf{R} Y$. The generic orbits meet $\mathfrak{h}^{\perp}$. Moreover, they meet in a 1-dimensional variety on which $H$ has open orbits. But there are infinitely many such orbits. This example was pointed out to me by $\mathrm{L}$. Corwin as an example wherein Theorem 1.1 fails for exponential solvable groups-i.e., we have the equal dimension situation but infinite multiplicity. Nevertheless, Theorem 2.2 still holds; i.e., we have the formula

$$
\operatorname{Ind}_{H}^{G} 1=\int_{\mathfrak{h}^{\perp} / H}^{\oplus} \pi_{\varphi} d \dot{\varphi} ;
$$

but unlike the nilpotent case the fibers of the map $\mathfrak{h}^{\perp} / H \rightarrow G \cdot \mathfrak{h}^{\perp} / G$ are infinite in number.

(iii) Abelian conormal coset spaces. Suppose $G=H V$ is a semidirect product, where $V$ is a closed normal vector subgroup of $G$. We make no assumption on $H$ other than $\hat{V} / H$ is countably separated. The homogeneous space $G / H$ is actually symmetric, and the representation $\operatorname{Ind}_{H}^{G} 1$ was decomposed in [16]. In fact

$$
\operatorname{Ind}_{H}^{G} 1=\int_{\hat{V} / H}^{\oplus} \pi_{\chi} d \dot{\chi},
$$

where $\pi_{\chi}=\operatorname{Ind}_{H_{\chi} V}^{G} \chi$ and $d \dot{\chi}$ is a pseudoimage of Lebesgue measure on $\hat{V}$ under $\hat{V} \rightarrow \hat{V} / H$. But the direct integral can be rewritten

$$
\operatorname{Ind}_{H}^{G} 1=\int_{\mathfrak{h}^{\perp} / H}^{\oplus} \pi_{\varphi} d \dot{\varphi}
$$

because $\mathfrak{h}^{\perp}$ is canonically and $H$-equivariantly identified to $\hat{V}$; for $\varphi \in \mathfrak{h}^{\perp}$, $\theta=\left.\varphi\right|_{\mathfrak{v}}, \chi(\exp X)=e^{i \theta(X)}, X \in \mathfrak{v}$, the subalgebra $\mathfrak{h}_{\theta}+\mathfrak{v}$ is a real polarization for $\varphi$; and $\pi_{\chi}$ is the representation associated to $\varphi$ by the orbit method.

(iv) Riemannian symmetric spaces. Let $G$ be a connected semisimple Lie group with finite center, $K$ a maximal compact subgroup. Let $G=K A N$ be an Iwasawa decomposition, $P=M A N$ the corresponding minimal parabolic subgroup. Then it is well known that

$$
\operatorname{Ind}_{H}^{G} 1=\int_{\hat{A} / W}^{\oplus} \pi_{\lambda} d \dot{\lambda}
$$

where

$$
\pi_{\lambda}=\operatorname{Ind}_{P}^{G} \chi_{\lambda}, \quad \chi_{\lambda}(\text { man })=\lambda(a), \lambda \in \hat{A},
$$


are the spherical principal series representations, $W=\operatorname{Norm}_{K}(A) / M A$ is the (finite) Weyl group, the decomposition is multiplicity-free, and $d \dot{\lambda}$ is the ordinar, image of Lebesgue measure $d \lambda$ on $\hat{A}$. (Actually one knows how to describe the intertwining operator and the precise measure in the class.) Let $\mathfrak{g}=\mathfrak{k}+\mathfrak{p}$ be the Cartan decomposition corresponding to $K$. Then $\mathfrak{k}^{\perp}$ is identified to $\mathfrak{p}$ via the Killing form. Let $\mathfrak{a}^{\prime}$ denote the regular elements in $\mathfrak{a} \subset \mathfrak{p}$. Then for every $\varphi \in \mathfrak{a}^{\prime}$, the minimal parabolic $m+\mathfrak{a}+\mathfrak{n}$ is a real polarization for $\varphi$, and the representation $\pi_{\varphi}$ associated to $\varphi$ by the orbit method is precisely $\pi_{\lambda}, \lambda(X)=e^{i\langle\varphi, X\rangle}, x \in \mathfrak{a}$. Moreover it is routine to check that $G \cdot \varphi \cap \mathfrak{p}=K \cdot \varphi$. Since $\mathfrak{p} / K=\hat{A} / W$, we have the formula of Theorem 2.2 once again, namely

$$
\operatorname{Ind}_{K}^{G} 1=\int_{\mathfrak{k}^{\perp} / K}^{\oplus} \pi_{\varphi} d \dot{\varphi} .
$$

(v) A non-Riemannian symmetric space. Let $G=\operatorname{SL}(2, R)$ and let $\sigma$ be the involution determined by the outer automorphism $g \rightarrow \varepsilon g \varepsilon^{-1}, \varepsilon=\left(\begin{array}{cc}1 & 0 \\ 0 & -1\end{array}\right)$. Then $H=G^{\sigma}=\left\{\left(\begin{array}{cc}a & 0 \\ 0 & a^{-1}\end{array}\right): a \neq 0\right\}$, a split Cartan subgroup. We have

$$
\operatorname{Ind}_{H}^{G} 1=\int_{-\infty}^{\infty} \pi_{t} \oplus \sum_{n \neq 0}^{\oplus} \pi_{n}
$$

where $\pi_{t}$ is the spherical principal series induced from the minimal parabolic $\left\{\left(\begin{array}{cc}a & b \\ 0 & a^{-1}\end{array}\right): a \neq 0, b \in \mathbf{R}\right\}$ by the unitary character

$$
\left(\begin{array}{cc}
a & b \\
0 & a^{-1}
\end{array}\right) \rightarrow|a|^{i t}
$$

and $\pi_{n}$ is the discrete series representation whose restriction to the maximal compact subgroup

$$
\left\{\left(\begin{array}{cc}
\cos \theta & \sin \theta \\
-\sin \theta & \cos \theta
\end{array}\right): 0 \leq \theta \leq 2 \pi\right\}
$$

has highest (resp. lowest) weight

$$
\left(\begin{array}{cc}
\cos \theta & \sin \theta \\
-\sin \theta & \cos \theta
\end{array}\right) \rightarrow \begin{cases}e^{-2 i n \theta}, & n>0 \\
e^{2 i n \theta}, & n<0\end{cases}
$$

The above representation is quasi-equivalent to $\operatorname{Ind}_{Z_{G}}^{G} 1, Z_{G}=\operatorname{Cent} G=$ $\left\{\left(\begin{array}{ll}\delta & 0 \\ 0 & \delta\end{array}\right): \delta^{2}=1\right\}$, the principal series occurs with multiplicity 2 , the discrete series with multiplicity 1 . Now, using the Killing form for identification, we have

$$
\mathfrak{h}^{\perp}=\left\{\left(\begin{array}{cc}
0 & \alpha \\
\beta & 0
\end{array}\right): \alpha, \beta \in \mathbf{R}\right\}
$$

the generic $G$-orbits passing through $\mathfrak{h}^{\perp}$ are parametrized by

$$
\left\{\left(\begin{array}{cc}
0 & \alpha \\
\alpha & 0
\end{array}\right): \alpha \neq 0\right\} \cup\left\{\left(\begin{array}{cc}
0 & \beta \\
-\beta & 0
\end{array}\right): \beta \neq 0\right\} ;
$$


the latter give rise to unitary representation iff $\beta$ is integral; and

$$
\begin{array}{ll}
G \cdot \varphi_{\alpha} \cap \mathfrak{h}^{\perp} & \text { has two components, } \\
G \cdot \varphi_{\beta} \cap \mathfrak{h}^{\perp} & \text { is connected. }
\end{array}
$$

Thus

$$
\operatorname{Ind}_{H}^{G} 1=\int_{\left(\mathfrak{h}^{\perp} \cap \mathscr{R}\right) / H}^{\oplus} \pi_{\varphi} d \dot{\varphi},
$$

where $\mathscr{R}$ denotes the regular integral orbits, and the measure $d \dot{\varphi}$ is Lebesgue or counting measure accordingly.

(vi) Nonexponential solvable groups. Let $\mathfrak{g}$ be the 3-dimensional Lie algebra with generators $W, X, Y$ satisfying relations

$$
[W, X]=Y, \quad[W, Y]=-X .
$$

Let $G=K V$ where $V=\exp (\mathbf{R} X+\mathbf{R} Y)$ is a normal vector subgroup, $K=$ $\exp \mathbf{R} W$ is a circle ( $G$ is the group of rigid motions of the plane). Let $H=$ $\exp \mathbf{R} X$. Then

$$
\begin{aligned}
\operatorname{Ind}_{H}^{G} 1 & =\operatorname{Ind}_{V}^{G} \operatorname{Ind}_{H}^{V} 1=\operatorname{Ind}_{V}^{G} \int^{\oplus} \chi_{(0, \eta)} d \eta \\
& =\int^{\oplus} \operatorname{Ind}_{V}^{G} \chi_{(0, \eta)} d \eta=2 \int^{\oplus} \pi_{\rho} d \rho,
\end{aligned}
$$

where $\pi_{\rho}=\operatorname{Ind}_{V}^{G} \chi,|\chi|=\rho . \operatorname{Ind}_{H}^{G} 1$ is quasi-equivalent to the regular representation of $G$ but has uniform multiplicity 2. $G / H$ is actually symmetric for the involution $\sigma: W \rightarrow-W, X \rightarrow X, Y \rightarrow-Y$. This and the previous example give instances of non-multiplicity-free symmetric spaces, which circumstance cannot occur for exponential solvable or Riemannian symmetric spaces. Even so, we have that every generic orbit meets $\mathfrak{h}^{\perp}$, and

$$
\# H \text {-orbits on } G \cdot \varphi \cap \mathfrak{h}^{\perp} \text { is } 2 \text {. }
$$

(vii) Oscillator algebras. Let $N$ be the Heisenberg group of dimension $2 n+$ 1 ; its Lie algebra $\mathfrak{n}$ has generators $X_{j}, Y_{j}, Z, j=1, \ldots, n$, satisfying $\left[X_{i}, Y_{j}\right]=\delta_{i j} Z$. Let $T$ be an $n$-torus acting on the Lie algebra level by

$$
\left[T_{i}, X_{j}\right]=\delta_{i j} Y_{j}, \quad\left[T_{i}, Y_{j}\right]=-\delta_{i j} X_{j} .
$$

$G=T N$ is an oscillator group, and we may consider $\operatorname{Ind}_{T}^{G} 1$. This representation is multiplicity-free; the generic functionals that occur in the spectrum are integral $\varphi \in \mathfrak{g}^{*}$ which satisfy $\varphi(Z)=\lambda \neq 0, \varphi\left(T_{j}\right)=-\operatorname{sgn}(\lambda) \omega_{j}, \omega_{j}$ a positive integer. In those cases we have $G \cdot \varphi \cap t^{\perp}=T \cdot \varphi$. Now we may modify the example in several interesting ways. Let $n=2$. Consider a single torus $T$ generated by an element $W$ which satisfies

$$
\left[W, X_{1}\right]=Y_{1}, \quad\left[W, Y_{1}\right]=-X_{1}, \quad\left[W, X_{2}\right]=-Y_{2}, \quad\left[W, Y_{2}\right]=X_{2} .
$$


Then $\operatorname{Ind}_{T}^{G} 1$ has uniform infinite multiplicity, and the corresponding $T$-orbits on the 2-dimensional variety $G \cdot \varphi \cap t^{\perp}$ are of dimension 1 . But if we change the relations to

$$
\left[W, X_{j}\right]=Y_{j}, \quad\left[W, Y_{j}\right]=-X_{j}, \quad j=1,2,
$$

then $\operatorname{Ind}_{T}^{G} 1$ has finite (unbounded) multiplicity, but the orbit dimensions are unchanged. That is, the multiplicity predicted by the geometry is infinite, whereas the actual multiplicity is finite.

\section{CONCLUSION}

The examples strongly suggest that the main results of Part I have applicability in a much wider realm than nilpotent or even exponential solvable groups. For simplicity let us continue to restrict attention to Theorem 2.2. Then the examples suggest the following: Let $G$ be a connected Lie group, $H$ a closed (almost) connected subgroup. Then

$$
\operatorname{Ind}_{H}^{G} 1=\int_{\left(\mathfrak{h}^{\perp} \cap \mathscr{R}\right) / H}^{\oplus} \pi_{\varphi} d \mu_{G, H}(\varphi)
$$

where $\mathscr{R}$ denotes the collection of orbits in $\mathfrak{g}^{*}$ that give rise to irreducible unitary representations-usually thought of as regular integral orbits, but actually admissible well-polarizable in the Duflo formulation [6]. How is formula (C) to be interpreted? The orbit method assigns representations to the functionals $\varphi \in \mathscr{R}$, but it may assign more than one. It depends on the size of the set $\mathscr{X}_{\varphi}\left([6,14]\right.$-roughly the number of characters of $G_{\varphi}$ which extend $\exp X \rightarrow e^{i \varphi(X)}, X \in \mathfrak{g}_{\varphi}$, from $\left.G_{\varphi}^{\circ}\right)$. In general, only those which are trivial on $G_{\varphi} \cap H$ will occur in $(\mathrm{C})$ - usually finitely many-and with the same multiplicity. The measure $\mu_{G, H}$ is somewhat problematic since the variety $\mathfrak{h}^{\perp} \cap \mathscr{R}$ may not be affine. But it will usually support a canonical measure, as recent work of Duflo and Vergne would indicate. Finally, the multiplicity in formula (C) is automatically

$$
\# H \text {-orbits in } G \cdot \varphi \cap \mathfrak{h}^{\perp}, \quad \varphi \in \mathfrak{h}^{\perp} \cap \mathscr{R} \text {. }
$$

Now while the spectra and measures thus predicted are almost certainly correct, the multiplicity formula may be incorrect-see example (vii). (Incidentally, one may construct other counterexamples, the simplest being $G$ connected compact, $H$ trivial; the $H$-orbits in the spheres $G \cdot \varphi=G \cdot \varphi \cap \mathfrak{h}^{\perp}$ are points, but of course the regular representation has finite multiplicity.) I do not know yet the modification of formula $(C)$ which is true in the greatest generality. But for (algebraic) exponential solvable groups, no modification is necessary. We shall see in a future publication that when a codimension 1 normal subgroup $N$ containing $H$ exists, we can generalize the arguments of Part I; and when it does not, we can generalize the arguments of Part II, $\S 7$. 


\section{REFERENCES}

1. L. Auslander and B. Kostant, Polarization and unitary representations of solvable Lie groups, Invent. Math. 14 (1971), 255-354.

2. L. Corwin and F. Greenleaf (with G. Grélaud), Direct integral decompositions and multiplicities for induced representations of nilpotent Lie groups, Trans. Amer. Math. Soc. 304 (1987), 549583.

3. Complex algebraic geometry and calculation of multiplicities for induced representations of nilpotent Lie groups, Trans. Amer. Math. Soc. 305 (1988), 601-622.

4. __ Spectrum and multiplicities for restrictions of unitary representations in nilpotent Lie groups, Pacific J. Math. 135 (1988), 233-267.

5. M. Duflo, Sur les extensions des représentations irréductibles des groupes de Lie nilpotents, Ann. Sci. École Norm. Sup. 5 (1972), 71-120.

6. Construction de représentations unitaires d'un groupe de Lie, Harmonic Analysis and Group Representations, C.I.M.E, 1982, pp. 129-222.

7. __ Théorie de Mackey pour les groupes de Lie algèbres, Acta Math. 149 (1982), 153-213.

8. G. Grélaud, Desintégration de représentations induites d'un groupe de Lie résoluble exponentiel, C.R. Acad. Sci. Paris 277 (1973), 327-330.

9. A. Kirillov, Unitary representations of nilpotent Lie groups, Russian Math. Surveys 17 (1962), 53-104.

10. A. Kleppner and R. Lipsman, The Plancherel formula for group extensions. I, Ann. Sci. École Norm Sup. 5 (1972), 459-576; II, 6 (1973), 103-132.

11. R. Lipsman, Characters of Lie groups II, J. Analyse Math. 31 (1977), 257-286.

12. __ Orbit theory and harmonic analysis on Lie groups with co-compact nilradical, J. Math. Pures Appl. 59 (1980), 337-374.

13. __ Orbit theory and representations of Lie groups with co-compact radical, J. Math. Pures Appl. 60 (1982), 17-39.

14. __ Harmonic induction on Lie groups, J. Reine Angew. Math. 344 (1983), 120-148.

15. __ Solvability of invariant differential operators with variable coefficients, Comm. Partial Differential Equations 10 (1985), 1261-1316.

16. __ Harmonic analysis on non-semisimple symmetric spaces, Israel J. Math. 54 (1986), 335350.

17. J. Milnor, Singular points of complex hypersurfaces, Ann. of Math. Studies, no. 61, Princeton Univ. Press, Princeton, N.J., 1968.

18. L. Pukanszky, On unitary representations of exponential groups, J. Funct. Anal. 2 (1968), 73-113.

19. __ Unitary representations of Lie groups with co-compact radical and applications, Trans. Amer. Math. Soc. 236 (1978), 1-50.

20. R. Strichartz, Harmonic analysis on Grassmanian bundles, Trans. Amer. Math. Soc. 296 (1986), 387-409.

21. M. Vergne, Étude de certaines représentations induites d'un groupe de Lie résoluble exponentiel, Ann. Sci. École Norm. Sup. 3 (1970), 353-384.

Department of Mathematics, University of Maryland, College Park, Maryland 20742 